\title{
The Great Observatories Origins Deep Survey
}

\section{VLT/ISAAC near-infrared imaging of the GOODS-South field $\star, \star \star$}

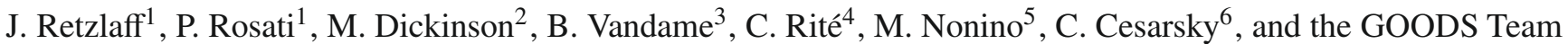 \\ ${ }^{1}$ European Organisation for Astronomical Research in the Southern Hemisphere (ESO), Karl-Schwarzschild-Str. 2, \\ 85748 Garching bei München, Germany \\ e-mail: jretzlaf@eso.org \\ 2 NOAO, 950 N. Cherry Avenue, Tucson, AZ 85719, USA \\ 3 Canon Research Centre, rue Touche Lambert, 35510 Cesson Sévigné, France \\ 4 Ministério da Ciência e Tecnologia - Observatório Nacional, Rua Gal. Jose Cristino 77, Sao Cristovao, Rio de Janeiro - RJ, Brasil \\ 5 INAF - Osservatorio Astronomico di Trieste, via Tiepolo 11, 34131 Trieste, Italy \\ ${ }^{6}$ CEA Saclay, Haute-commissaire à l’Énergie Atomique, 91191 Gif-sur-Yvette, France
}

Received 20 July 2009 / Accepted 7 December 2009

\section{ABSTRACT}

\begin{abstract}
Aims. We present the final public data release of the VLT/ISAAC near-infrared imaging survey in the GOODS-South field. The survey covers an area of $172.5,159.6$ and $173.1 \mathrm{arcmin}^{2}$ in the $J, H$, and $K_{\mathrm{s}}$ bands, respectively. For point sources total limiting magnitudes of $J=25.0, H=24.5$, and $K_{\mathrm{s}}=24.4(5 \sigma, \mathrm{AB})$ are reached within $75 \%$ of the survey area. Thus these observations are significantly deeper than the previous EIS Deep Public Survey which covers the same region. The image quality is characterized by a point spread function ranging between $0.34^{\prime \prime}$ and $0.65^{\prime \prime} F W H M$. The images are registered to a common astrometric grid defined by the GSC 2 with an accuracy of $\sim 0.06^{\prime \prime}$ RMS over the whole field. The overall photometric accuracy, including all systematic effects, adds up to 0.05 mag. The data are publicly available from the ESO science archive facility.

Methods. We describe the data reduction, the calibration, and the quality control process. The final data set is characterized in terms of astrometric and photometric properties, including the PSF and the curve of growth. We establish an empirical model for the sky background noise in order to quantify the variation of limiting depth and statistical photometric errors over the survey area. We define a catalog of $K_{\mathrm{s}}$-selected sources which contains $J H K_{\mathrm{s}}$ photometry for 7079 objects. Differential aperture corrections were applied to the color measurements in order to avoid possible biases as a result of the variation of the PSF. We briefly discuss the resulting color distributions in the context of available redshift data. Furthermore, we estimate the completeness fraction and relative contamination due to spurious detections for source catalogs extracted from the survey data. For this purpose, an empirical study based on a deep $K_{\mathrm{s}}$ image of the Hubble Ultra Deep Field is combined with extensive image simulations.

Results. With respect to previous deep near-infrared surveys, the surface density of faint galaxies has been established with unprecedented accuracy by virtue of the unique combination of depth and area of this survey. We derived galaxy number counts over eight magnitudes in flux up to $J=25.25, H=25.0, K_{\mathrm{s}}=25.25$ (in the AB system). Very similar faint-end logarithmic slopes between 0.24 and $0.27 \mathrm{mag}^{-1}$ were measured in the three bands. We found no evidence for a significant change in the slope of the logarithmic galaxy number counts at the faint end.
\end{abstract}

Key words. cosmology: observations - large-scale structure of the Universe - galaxies: evolution - infrared: galaxies - surveys

\section{Introduction}

The Great Observatories Origins Deep Survey (GOODS) aims at combining the best and deepest data from X-ray through radio wavelengths and making them publicly available to the community. The combined data enable studies of the distant universe in terms of the formation and evolution of galaxies and active galactic nuclei, the distribution of dark and

^ Based on observations using the Very Large Telescope at the Paranal Observatory of the European Organisation for Astronomical Research in the Southern Hemisphere, Chile, under ESO programmes 168.A-0485, 64.O-0643, 66.A-0572, 68.A-0544, and 73.A0764 .

$\star \star$ Reduced data products presented in this publication, including calibrated images and the $J H K_{\mathrm{s}}$ source catalog, can be retrieved from the ESO science archive facility via the http://archive.eso.org/cms/eso-data/data-packages/ goods-isaac-final-data-release-version-2-0 luminous matter at high redshift and the origin of extragalactic background radiation. Observations are centered on the two target fields: the Hubble Deep Field North and the Chandra Deep Field South (CDF-S), each of them covering an area of $160 \mathrm{arcmin}^{2}$. The data are obtained using the most powerful facilities in space and on the ground, i.e. the NASA Great Observatories, the Spitzer Space Telescope, Hubble (HST), and Chandra, ESA's XMM-Newton, the 8.2 m-aperture Very Large Telescopes at the ESO Paranal Observatory, the twin 10-m Keck Telescopes, the 8.2-m Subaru Telescope, and the 8-m telescopes of the Gemini Observatory. For an overview of the GOODS project, see Dickinson et al. (2003), Giavalisco et al. (2004), and Giavalisco (2005).

To study the evolution of galaxies, selection at near-infrared (NIR) wavelengths has a number of advantages over optical selection, the most significant one being the small dependence on type out to redshifts of about three (e.g. Cowie et al. 1994). The discovery of a population of distant galaxies based on NIR color 
selection (see, e.g., Franx et al. 2003; Daddi et al. 2004) which would have been missed by the Lyman break technique demonstrates the importance of deep NIR imaging for the understanding of the history of mass assembly in the universe.

While deep ground-based optical imaging has become largely obsolete due to the GOODS ACS data set, high-quality NIR observations had to be executed from a ground-based facility because the small field of view of NICMOS on HST would have made NIR imaging of the GOODS region overly costly. However, NIR imaging remains a key requirement for the scientific goals of the GOODS program because sampling of the NIR spectral range is crucial if spectral energy distributions have to be fitted in order to derive accurate photometric redshifts and stellar population parameters of galaxies. ESO has dedicated a substantial amount of observing time in order to support the community with imaging and also VLT spectroscopy for the target field CDF-S (Renzini et al. 2003).

There were four public incremental releases of the GOODS ISAAC data: version 0.5 (April 2002), version 1.0 (April 2004), version 1.5 (September 2005), and version 2.0 (September 2007). In this publication we present the final version (2.0) of the fully reduced and calibrated images which have been obtained with the Infrared Spectrometer And Array Camera (ISAAC) in the $J, H$, and $K_{\mathrm{s}}$ bands. This data set was released via the ESO science archive facility as part of the ESO/GOODS project.

The paper is organized as follows. Section 2 outlines the observations, Sect. 3 describes the image data reduction and calibration process. The final survey images, their properties and an assessment of the photometric calibration are given in Sect. 4. In Sect. 5, we describe how photometric source catalogs were defined and discuss their characteristics in term of color-magnitude and color-color diagrams. In Sect. 6, first, catalog completeness and contamination is discussed, and thereafter deep galaxy number counts in the three survey bands are presented. Finally, we conclude in Sect. 7.

Note that throughout this work magnitudes are expressed in the AB photometric system (Oke \& Gunn 1983) unless otherwise noted.

\section{Observations}

The Infrared Spectrometer and Array Camera (ISAAC) mounted on the first of the four 8.2-m VLT unit telescopes at Cerro Paranal is equipped with a $1024 \times 1024$ pixels $\mathrm{HgCdTe}$ Rockwell Hawaii array having a pixel scale of $0.148^{\prime \prime}$, corresponding to a $2.5^{\prime} \times 2.5^{\prime}$ field of view (Moorwood et al. 1999). Figure 1 shows how the ISAAC pointings were laid out to assemble a mosaic of the CDF-S region. A contiguous area of 24 fields, $150 \operatorname{arcmin}^{2}$ respectively, are covered in the $J, H$, and $K_{\mathrm{s}}$ bands, plus 2 additional fields (F01 and F02) at the top of the survey area which have $J$ and $K_{\mathrm{s}}$ but no $H$ data. In the early stages of the project, in an attempt to maximize the overall survey efficiency, fields F25 and 26 were re-arranged and replaced with F25n and 26n. The $K_{\mathrm{s}}$ band data which was obtained for fields F25 and 26 has been included in the survey data processing in the same way as the other fields despite their shallowness. Later on, this auxiliary data proved to be very valuable for the purpose of validation of the internal photometric consistency (Sect. 4.3). See Table 1 for the field coordinates.

The GOODS ESO ISAAC program was originally planned to reach $5 \sigma$ limiting magnitudes of $25.2,24.7$, and 24.4 in $J$, $H$, and $K_{\mathrm{s}}$, respectively, in order to roughly match pre-launch expectations for Spitzer IRAC sensitivity at 3.6 to $8 \mu \mathrm{m}$. The

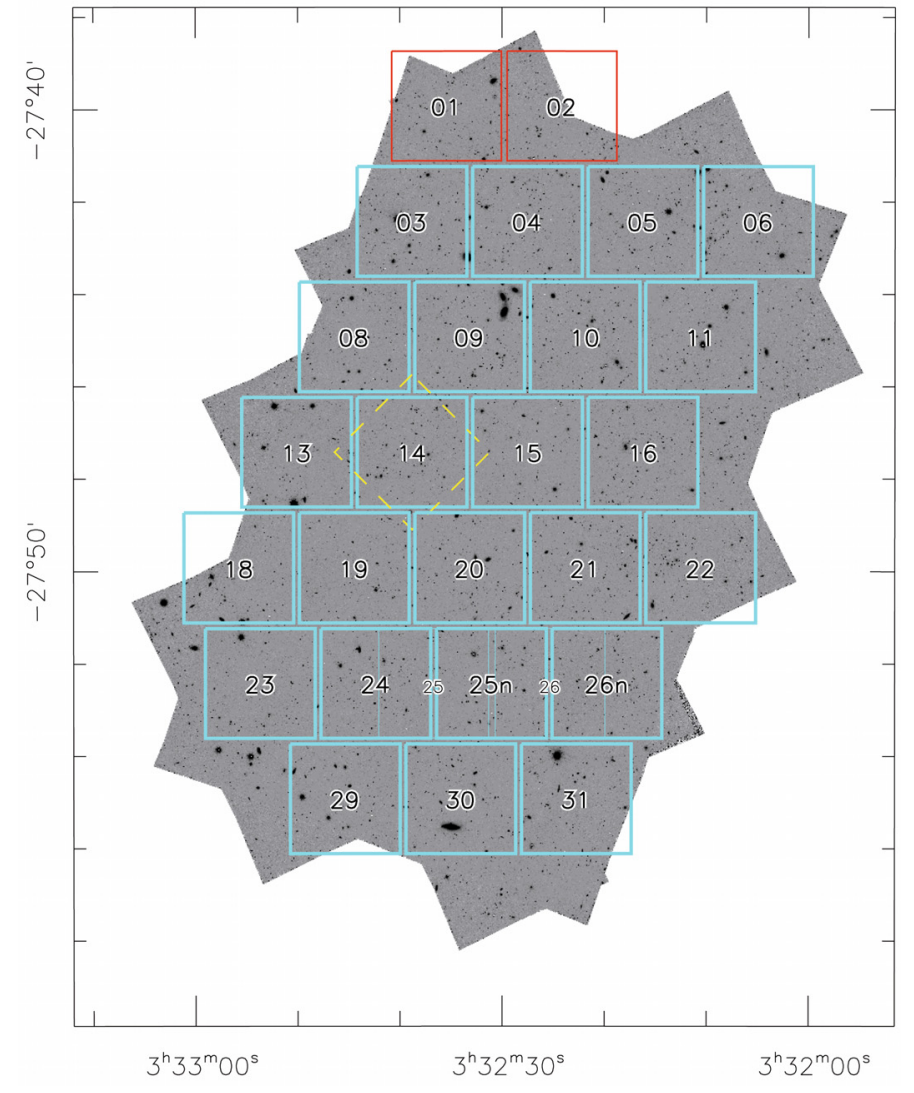

Fig. 1. Tiling of the CDF-S region with $2.5^{\prime} \times 2.5^{\prime}$ ISAAC fields displayed over the HST/ACS GOODS $z$-band mosaic (Giavalisco et al. 2004). The fields on the very top, F01 and F02, are covered in $J$ and $K_{\mathrm{s}}$, the other 24 fields (F03 through F31) in all three bands $J, H$, and $K_{\text {s. }}$. The dashed diamond centered on field F14 is the footprint of the deep $\mathrm{UDF} / \mathrm{ISAAC} K_{\mathrm{s}}$ image.

intent was to provide NIR flux measurements for the large majority of IRAC-detected galaxies, as well as images with higher angular resolution in order to facilitate the deblending of sources in crowded regions of the lower-resolution IRAC data. In practice, Spitzer IRAC in-flight performance at 3.6 and $4.5 \mu \mathrm{m}$ significantly exceeded the pre-launch expectations, making the GOODS data at those wavelengths substantial deeper than any ground-based NIR program could reasonably match. Given the instrumental sensitivity, a total integration time per tile of 3.5, 5, and $6 \mathrm{~h}$ was estimated to reach the intended depth. To achieve accurate sky background subtraction which is most crucial for deep NIR imaging, the commonly practiced jitter imaging technique was used. A jitter box size of $25^{\prime \prime}$ was chosen within which the control system automatically offsets the telescope between subsequent integrations. Detector integration times (DITs) between 10 and $30 \mathrm{~s}$ were used, according to the typical sky brightness (darker at $J$, brighter at $H$ and $K_{\mathrm{s}}$ ). The number of detector integrations (NDIT) was chosen so that total integration times $($ DIT $\times$ NDIT) between 60 and $180 \mathrm{~s}$ were used. The survey was executed in observation blocks (OBs) each of which comprising the integration of a single field in one filter for a total integration time of normally $3600 \mathrm{~s}$ or $1800 \mathrm{~s}$, and, thus, resulting in between 15 and 40 images. The total actual integration time of the data that were combined in the final survey images amounts to $360 \mathrm{~h}$. Given that some data were discarded and taking into account observing overheads the whole program was allocated about $500 \mathrm{~h}$ of observing time. The observations have been 
Table 1. Nominal center positions of the GOODS/ISAAC survey fields.

\begin{tabular}{|c|c|c|}
\hline Field & $\begin{array}{c}\text { RA } \\
(\mathrm{J} 2000)\end{array}$ & $\begin{array}{c}\text { Dec } \\
(\mathrm{J} 2000) \\
\end{array}$ \\
\hline F01 & $3^{\mathrm{h}} 32^{\mathrm{m}} 35^{\mathrm{s}} .4$ & $-27^{\circ} 39^{\prime} 55^{\prime \prime}$ \\
\hline F02 & $3^{\mathrm{h}} 32^{\mathrm{m}} 24^{\mathrm{s}} .1$ & $-27^{\circ} 39^{\prime} 55^{\prime \prime}$ \\
\hline F03 & $3^{\mathrm{h}} 32^{\mathrm{m}} 38^{\mathrm{s}} .8$ & $-27^{\circ} 42^{\prime} 25^{\prime \prime}$ \\
\hline F04 & $3^{\mathrm{h}} 32^{\mathrm{m}} 27^{\mathrm{s}} .5$ & $-27^{\circ} 42^{\prime} 25^{\prime \prime}$ \\
\hline F05 & $3^{\mathrm{h}} 32^{\mathrm{m}} 16^{\mathrm{s}} .2$ & $-27^{\circ} 42^{\prime} 25^{\prime \prime}$ \\
\hline F06 & $3^{\mathrm{h}} 32^{\mathrm{m}} 4^{\mathrm{s}} .9$ & $-27^{\circ} 42^{\prime} 25^{\prime \prime}$ \\
\hline F08 & $3^{\mathrm{h}} 32^{\mathrm{m}} 44^{\mathrm{s}} .4$ & $-27^{\circ} 44^{\prime} 55^{\prime \prime}$ \\
\hline F09 & $3^{\mathrm{h}} 32^{\mathrm{m}} 33^{\mathrm{s}} .1$ & $-27^{\circ} 44^{\prime} 55^{\prime \prime}$ \\
\hline F10 & $3^{\mathrm{h}} 32^{\mathrm{m}} 21^{\mathrm{s}} .8$ & $-27^{\circ} 44^{\prime} 55^{\prime \prime}$ \\
\hline F11 & $3^{\mathrm{h}} 32^{\mathrm{m}} 10^{\mathrm{s}} .5$ & $-27^{\circ} 44^{\prime} 55^{\prime \prime}$ \\
\hline F13 & $3^{\mathrm{h}} 32^{\mathrm{m}} 50^{\mathrm{s}} .1$ & $-27^{\circ} 47^{\prime} 25^{\prime \prime}$ \\
\hline F14 & $3^{\mathrm{h}} 32^{\mathrm{m}} 38^{\mathrm{s}} .8$ & $-27^{\circ} 47^{\prime} 25^{\prime \prime}$ \\
\hline F15 & $3^{\mathrm{h}} 32^{\mathrm{m}} 27.5$ & $-27^{\circ} 47^{\prime} 25^{\prime \prime}$ \\
\hline F16 & $3^{\mathrm{h}} 32^{\mathrm{m}} 16^{\mathrm{s}} .2$ & $-27^{\circ} 47^{\prime} 25^{\prime \prime}$ \\
\hline F18 & $3^{\mathrm{h}} 32^{\mathrm{m}} 55^{\mathrm{s}} .7$ & $-27^{\circ} 49^{\prime} 55^{\prime \prime}$ \\
\hline F19 & $3^{\mathrm{h}} 32^{\mathrm{m}} 44^{\mathrm{s}} .4$ & $-27^{\circ} 49^{\prime} 55^{\prime \prime}$ \\
\hline F20 & $3^{\mathrm{h}} 32^{\mathrm{m}} 33^{\mathrm{s}} .1$ & $-27^{\circ} 49^{\prime} 55^{\prime \prime}$ \\
\hline F21 & $3^{\mathrm{h}} 32^{\mathrm{m}} 21^{\mathrm{s}} .8$ & $-27^{\circ} 49^{\prime} 55^{\prime \prime}$ \\
\hline F22 & $3^{\mathrm{h}} 32^{\mathrm{m}} 10^{\mathrm{s}} .5$ & $-27^{\circ} 49^{\prime} 55^{\prime \prime}$ \\
\hline F23 & $3^{\mathrm{h}} 32^{\mathrm{m}} 53^{\mathrm{s}} .6$ & $-27^{\circ} 52^{\prime} 25^{\prime \prime}$ \\
\hline F24 & $3^{\mathrm{h}} 32^{\mathrm{m}} 42^{\mathrm{s}} \cdot 3$ & $-27^{\circ} 52^{\prime} 25^{\prime \prime}$ \\
\hline F25 & $3^{\mathrm{h}} 32^{\mathrm{m}} 36^{\mathrm{s}} .7$ & $-27^{\circ} 52^{\prime} 25^{\prime \prime}$ \\
\hline$F 25 n$ & $3^{\mathrm{h}} 32^{\mathrm{m}} 31^{\mathrm{s}} .0$ & $-27^{\circ} 52^{\prime} 25^{\prime \prime}$ \\
\hline F26 & $3^{\mathrm{h}} 32^{\mathrm{m}} 25^{\mathrm{s}} \cdot 3$ & $-27^{\circ} 52^{\prime} 25^{\prime \prime}$ \\
\hline F26n & $3^{\mathrm{h}} 32^{\mathrm{m}} 19^{\mathrm{s}} .7$ & $-27^{\circ} 52^{\prime} 25^{\prime \prime}$ \\
\hline F29 & $3^{\mathrm{h}} 32^{\mathrm{m}} 45^{\mathrm{s}} .3$ & $-27^{\circ} 54^{\prime} 55^{\prime \prime}$ \\
\hline F30 & $3^{\mathrm{h}} 32^{\mathrm{m}} 34^{\mathrm{s}} .0$ & $-27^{\circ} 54^{\prime} 55^{\prime \prime}$ \\
\hline F31 & $3^{\mathrm{h}} 32^{\mathrm{m}} 22^{\mathrm{s}} .7$ & $-27^{\circ} 54^{\prime} 55^{\prime \prime}$ \\
\hline
\end{tabular}

executed in service mode in 216 nights between October 1999 and January 2007.

The data were obtained under the ESO large programme 168.A-0485, led by Cesarsky, in direct support to the GOODS project. Data covering four ISAAC fields in $J$ and $K_{\mathrm{s}}$ bands were also drawn from the ESO programmes 64.O-0643, 66.A-0572 and 68.A-0544, led by Giallongo (see Saracco et al. 2001).

\section{Image data reduction and calibration}

\subsection{Overview}

The overall survey data reduction and calibration process is schematically illustrated in Fig. 2. To begin with, the ESO/MVM data reduction system was used to process the raw jitter mode observations using the required calibration files to correct for instrumental signatures and to produce $O B$ images, that is one co-added image with associated weight map per OB being astrometrically registered to the predefined astrometric grid of the survey. The fully automated processing of the entire data set of 13964 raw jitter mode frames and 20699 calibration files resulted in 426 astrometrically calibrated OB images (Sects. 3.2, 3.3). Then, the OB images were characterized in terms of PSF width, photometric uniformity, and depth in order to identify and remove images of low-quality which would have degraded the quality of the final survey products (Sect. 3.4).

For the photometric calibration of the OB images, at first, the raw standard star observations were reduced to instrumental photometric zero points using the master flatfield calibrations generated by ESO/MVM. From this data set, a subset of "best" zero points was constructed. Then, photometric scalings of OB

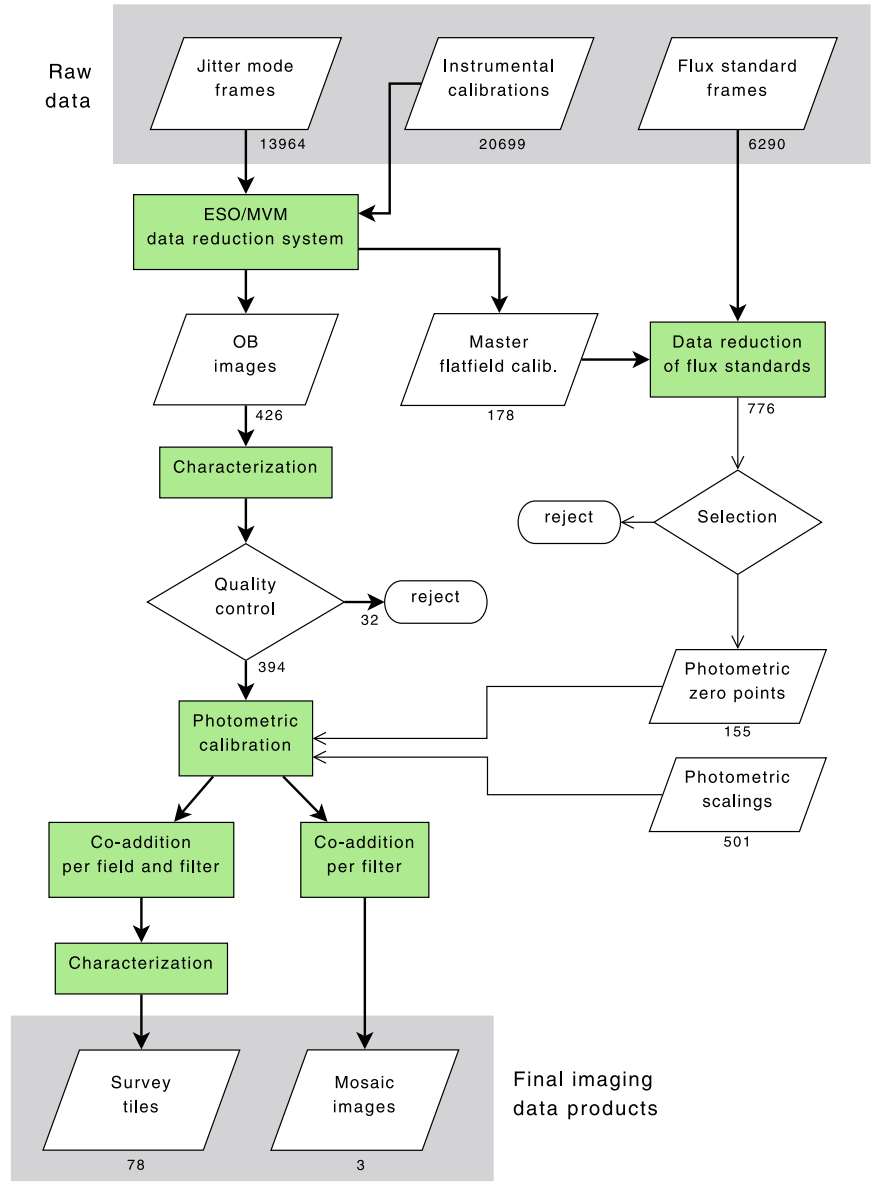

Fig. 2. Schematic flow of the data reduction and calibration process from raw data to final, fully calibrated survey data products (from top to bottom). Processing steps are represented by rectangles, input/output data by parallelograms, and thick arrows indicate the image data flow. Numbers represent the cardinality of each data set. Refer to the text for a detailed description.

images belonging to the same tile and between adjacent tiles were measured and combined with the zero points to determine - in a robust way - an accurate photometric calibration for all OB images equalized over the whole survey field (Sect. 3.5). That followed, the photometrically calibrated OB images were co-added to produce the survey tiles which subsequently underwent a final step of image quality characterization.

\subsection{Data processing}

The ESO/MVM software, version 1.3.4, has been used for image data reduction. ESO/MVM follows well established procedures for the reduction of NIR imaging observations taken in jitter mode by featuring a two-pass scheme to mask out sources prior to the final background estimation. In the following, we will outline the main reduction steps executed by ESO/MVM. Comprehensive documentation of ESO/MVM including all the reduction steps and algorithmic details being implemented is beyond the scope of this publication and may be found in Vandame (2004) which serves as reference documentation and describes in 
length the implemented algorithms, parameter settings, instrument specific configurations, and sample applications ${ }^{1}$.

First, the raw calibration data, namely dark frames and twilight sky flats, were combined into master calibration files and used to correct the raw scientific images for the basic instrumental signatures. No attempt was made to correct for detector nonlinearity. However, the overall effect is expected to be rather small because the scientific OBs for the survey program and, by the same token, the OBs for photometric standards had been generally designed with the objective to operate the detector in the linear regime under nominal conditions. The magnitude of possible nonlinearity effects on the final results is discussed in Sect. 3.5.3.

In a few cases, overly long OBs were split in two blocks, or, particularly short, partially completed, OBs were merged prior to data reduction in order to optimize the quality of the reduction. Sky background images were computed from groups of between 13 and 15 consecutive jitter images (depending on filter) using sigma-clipped pixel-by-pixel image combination. Each science image was sky-subtracted using the linear interpolation in time of the two corresponding successive sky background images. Possible transparency variations from image to image were monitored by means of the signal-to-noise ratio (SNR) based on which outliers with exceptionally low SNR were automatically discarded. An individual rescaling of the images has not been done.

Then, for each $\mathrm{OB}$, the individual background-subtracted images were astrometrically registered to each other with subpixel accuracy and co-added (see below) to form a preliminary version of the $\mathrm{OB}$ images. The purpose of these first-pass images is the creation of masks that mask the astronomical sources in order to improve the sky background computation in the second pass. To this end all sources exceeding $\sim 3$ times the local RMS noise were detected on the PSF-convolved image and - to also exclude the wings of the source profile - each source's region was artificially enlarged by a factor of 2 (linearly) before creating its mask. Thus, in the second pass, the sky background images were re-computed exclusively from image pixels that belong to background regions whereas the rest of the procedure was repeated unaltered.

\subsection{Astrometric calibration}

The astrometric calibration is based on a dense reference catalog which was generated by the GOODS team from a deep $R$-band image of the CDF-S and was also used for the production of the GOODS/ACS image mosaics (Giavalisco et al. 2004). The image was obtained with the Wide Field Imager mounted at the 2.2-m MPG/ESO telescope at La Silla, and astrometrically calibrated using the Guide Star Catalog (GSC 2). Each OB image of the GOODS/ISAAC survey was astrometrically registered using the reference catalog whereas image distortions were modeled with a $3^{\text {rd }}$ order polynomial. This resulted in an internal astrometric accuracy between $0.05^{\prime \prime}$ and $0.06^{\prime \prime}$ RMS as measured for sources brighter than 20 mag in the final mosaic images in all three filters. The relative registration between bands is accurate to $\sim 0.03^{\prime \prime}$.

For the astrometric grid of the $\mathrm{OB}$ images the same projection as for the GOODS/ACS images has been adopted and a pixel size of $0.15^{\prime \prime}$ has been chosen so that one GOODS/ISAAC

\footnotetext{
1 The full software package may be downloaded from http://archive.eso.org/cms/eso-data/data-packages/ eso-mvm-software-package
}

pixel subtends exactly a block of $5 \times 5$ GOODS/ACS pixels. The individual jitter images were resampled to this grid using the Lanczos-3 interpolation kernel. Pixel-to-pixel noise correlation, the interpolation process has introduced, is quantified in Sect. 3.4. During the process of image co-addition, badpixel masks were taken into account and each contribution was recorded pixel-by-pixel to build up respective weight maps.

The comparison of the final mosaics with the calibrated data from the Hubble Space Telescope (HST) Advanced Camera for Surveys (ACS) in its version 1.1 incarnation yields astrometric offsets of typically $\sim 0.1^{\prime \prime}$ RMS across the entire area which can be attributed primarily to zonal residuals that are known to be present in the astrometric calibration of the HST/ACS mosaics.

\subsection{Quality control of $O B$ images}

For each OB image the quality was quantified in terms of the $F W H M$ of stellar sources ("seeing"), the sky noise as a function of aperture size, and the photometric scaling of images belonging to the same tile and filter. Then, based on the inspection of these quality parameters with respect to the whole sample, images of significantly low quality were identified and sorted out, followed by a visual inspection, to ensure that no corrupted image slipped through.

In order to determine the average $F W H M$ of stellar sources, we made use of a list of ca. 400 sources that appear point-like in the GOODS/ACS $z$-band image. Based on this list we preselected appropriate candidate sources for the PSF characterization.

The sky noise was measured for each OB image in a similar fashion as described by Labbé et al. (2003), that is, first, a noiseequalized image was created by multiplication with the square root of the weight map. Then, the image was segmented into background and sources using a $\sim 2.6 \sigma$ significance threshold plus a 5 pixel wide safety margin around each source segment. Then, the background flux was sampled with non-overlapping circular apertures with diameters between $0.5^{\prime \prime}$ and $4.0^{\prime \prime}$ and a linear relation of the form $(a+b s) s$ was fitted to the dispersion to obtain the two parameters $a$ and $b$. Here, the linear aperture size, $s$, is defined as the square root of the aperture area in pixel units. It has been verified that the adopted parameterization describes the actual data adequately. Therewith, the sky noise, that is the statistical fluctuation of the flux measured within an aperture of size $s$ at any point of the image can be expressed by the Gaussian dispersion

$\sigma_{\text {sky }}(s)=\frac{\sigma_{0}}{\sqrt{w_{j}}}(a+b s) s$,

where $\sigma_{0}$ is the RMS of all background image pixels, and $w_{j}$ is the relative weight ${ }^{2}$ of pixel $j$. This empirical model of the sky noise accounts for the pixel-to-pixel correlation which results from the preceding image interpolation step and which breaks the simple scaling with aperture scale $\sigma_{\text {sky }} \propto s$. According to the interpolation kernel, the correlation length is not more than a few pixels. To quantify the amount of this small-scale contribution, the total $a+b$ is typically about 1.15 , that is, at the

\footnotetext{
2 For the relative weight, $w_{j}$, a normalization to unity at the median pixel has been adopted, i.e., $w_{j} \equiv W_{j} /$ median $\left(W_{j}\right)$ with $W_{j}$ denoting the inverse variance weight map and the median being taken of all pixels of the given image having $W_{j}>0$. Thus, $\sigma_{0}$ refers to the median pixel. This notion, using the index zero, has been adopted throughout the text for quantities scaling with the weight map, namely the limiting depth and the gain conversion factor.
} 


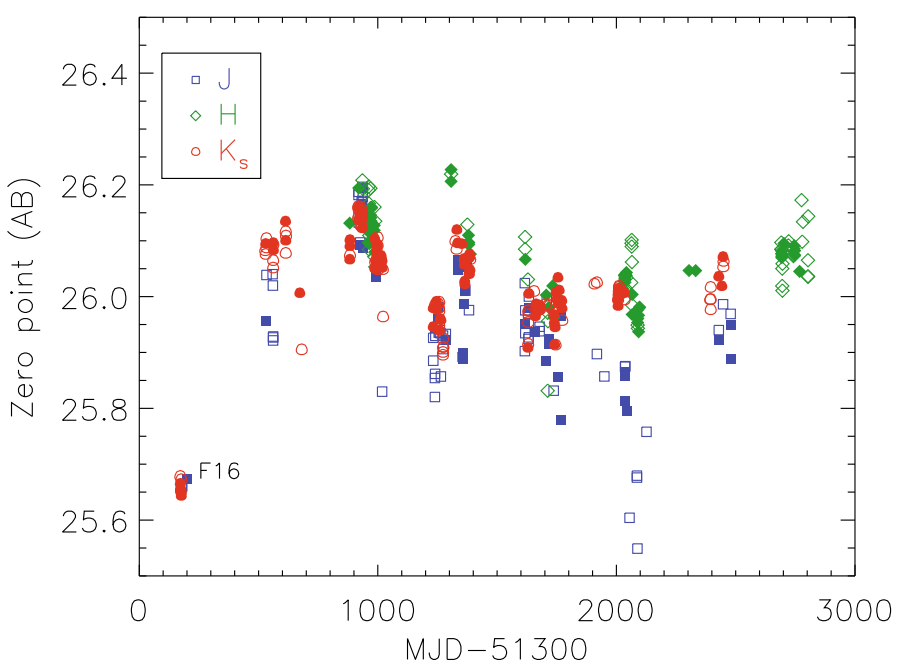

Fig. 3. Variation of the photometric zero point of the GOODS/ISAAC OB images from October 1999 until January 2007. Filled symbols correspond to OBs with flux standard star calibrations, open symbols refer to $\mathrm{OBs}$ whose zero points have been inferred by photometric scaling. Zero points have been converted to unit airmass to produce this plot. However, this correction is negligibly small compared to the gross variation.

scale of a single pixel, i.e. $s=1$, the direct estimation $\sigma_{0}$ underestimates the flux dispersion by about $15 \%$. The other contribution which affects slightly larger scales is due to imperfect background subtraction, that is residual spatial modulations of the image background. If the night sky background is highly variable with a time scale being shorter than the one implicit to the averaging process that is part of the image data reduction, then such residuals may remain - mostly apparent in the form of ripples or speckles. Not surprisingly, the parameters $a$ and $b$ are sensitive indicators for the presence of such residuals. Therefore, we have employed them to identify and reject $O B$ images which were manifestly affected above average.

In the course of the quality control process, $15 \mathrm{OB}$ images were rejected because of the seeing being worse than $0.85^{\prime \prime}$ $F W H M$, nine for being very noisy and showing particularly strong residuals in the sky background $(b>0.15)$, five for being exceptionally "shallow" with the photometric zero point of more than 0.3 mag below average, and three for other reasons such as corrupted data. In total, 394 OB images, which account for $93.7 \%$ of the total integration time of reduced OBs, passed the quality control criteria and underwent the following steps of photometric calibration, image stacking, and final characterization which we will describe in the next section.

\subsection{Photometric calibration}

\subsubsection{Photometric zero points}

As the GOODS/ISAAC program was spread over a large time frame, the substantial variation of the instrumental photometric zero point on various time scales is not surprising (Fig. 3). These variations are caused by instrumental interventions, the steady degradation of the mirrors' reflectivity, mirror re-coating events, and, finally, the variation of atmospheric transparency. The significantly low instrumental zero point at the beginning of VLT operations, for instance, when the $J$ and $K_{\mathrm{s}}$ data for tile F16 were being obtained, is likely due to accumulated dust on the mirror of UT1 caused by the ongoing construction of the other UTs at that time. In order to establish an accurate and consistent photometric calibration across the whole survey area, we have combined photometric zero points (ZP) obtained from standard stars with relative photometric scalings between survey tile images.

Instrumental ZPs were obtained using faint NIR standard stars which are being observed on a nightly basis as part of the observatory's instrument calibration plan. Most of the flux standards were drawn from Persson et al. (1998) and Hunt et al. (1998), complemented by a small number of UKIRT standards (Hawarden et al. 2001).

The available data of photometric flux standard observations was carefully selected to minimize the potential bias due to detector nonlinearity. For each image of a photometric standard star the peak signal including the background was used as quality indicator and outliers exceeding 15000 ADU which corresponds to a formal nonlinearity of $1 \%$ (Amico et al. 2002) were eliminated. The remaining set of photometric calibrations from which the ZPs were finally determined is dominated to $85 \%$ by data with peak fluxes below $10000 \mathrm{ADU}$ which corresponds to a formal nonlinearity of ca. $0.5 \%$ while the remaining $15 \%$ of the data have peak fluxes above 10000 ADU. Additionally, we have examined more than 5000 individual photometric calibrations in $J$, $H$, and $K_{\mathrm{s}}$ band as a function of the maximum signal level to empirically check for a possible systematic trend in the derived ZPs. Up to a signal level of 15000 ADU, we found no evidence that ZPs are systematically biased low. Merely beyond 20000 ADU, i.e. for data that was finally not used for calibration, a decline by $\gtrsim 0.03$ mag becomes apparent in all three bands. Hence, we can conclude that the ZPs which were eventually used to anchor the survey's photometry are affected by detector nonlinearity by less than 0.01 mag even in the worst case.

We started out by selecting all the standard star observations within an interval of plus or minus 2 nights around each science observation and derived a total of 776 zero points - by far more than what went into the final photometric solution (see below). ZPs were computed from the instrumental flux measured within an aperture of $10^{\prime \prime}$ diameter in compliance with Persson et al. (1998).

Since flux standards had not always been observed immediately before or after each science integration, we adopted the following scheme for the association of ZPs aiming at a practical compromise between conservatively minimizing the effect of possible transparency variations and ending up with a sufficient number of ZPs. For each OB the ZP whose flux standard had been acquired closest in time to the science observation within a time interval of less than $2 \mathrm{~h}$ and an airmass difference below 0.5 was selected. If these conditions were not met by any ZP, the respective OB was not assigned a ZP but the photometric calibration was purely inferred from the scaling relative to other OBs belonging to the same or to neighboring tiles (see below). Using these criteria, 210 out of the 394 OB images were associated with $165 \mathrm{ZPs}, 50 \%$ of which were obtained in a time interval of plus or minus 45 min with respect to the corresponding science observation.

Then, we have inspected each associated ZP in the context of all the other ZPs obtained within an interval of 5 nights so as to identify non-photometric conditions and, hence, we have dismissed 10 ZPs for being low by $\gtrsim 0.05 \mathrm{mag}$.

The resulting 155 instrumental ZPs were converted into ZPs for 199 OB images using the atmospheric extinction coefficients for the ISAAC instrument, 0.09, 0.04, and 0.06 mag per unit airmass for $J, H$, and $K_{\mathrm{s}}$, respectively, as determined by Mason et al. (2008).

The match between the ISAAC filters $\left(J, H\right.$, and $\left.K_{\mathrm{S}}\right)$ and those used to establish the faint IR standard star system of 
Persson et al. (1998) is quite good and one expects color terms which differ from 0 by less than 0.01 (Amico et al. 2002). As the color transformation between ISAAC magnitudes and those of LCO have never been experimentally verified, and our data does not allow for it either, we have chosen not to apply any color correction.

\subsubsection{Global photometric calibration}

To determine a survey-wide photometric solution per filter, photometric scalings between individual OB images were computed. As it was straightforward to determine the relative flux scaling for images belonging to the same tile and filter with sub per-cent accuracy once PSF matching had been done, the scaling for neighboring tiles turned out to be difficult to measure, because, usually, the overlapping area was too small to find a sufficient number of high signal-to-noise sources for accurate photometry. Therefore, we have employed public data from the ESO science archive which covers the survey area in the same bands in order to establish accurate relative photometric calibrations between adjacent survey fields. The data had been obtained using the SOFI instrument (Moorwood et al. 1998) mounted on the New Technology Telescopy at La Silla. SOFI's field of view of about $4.9^{\prime} \times 4.9^{\prime}$ guarantees sufficient overlap between ISAAC and SOFI images in general, while other instrument characteristics are similar by construction, in particular, the instrumental response. The $J$ and $K_{\mathrm{s}}$ observations that were used belong to the infrared part of the ESO Imaging Survey (e.g. Olsen et al. 2006), the $H$ band data were originally obtained within a pro-

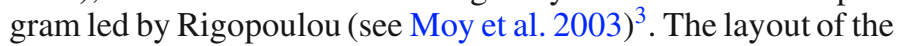
$J H K_{\mathrm{S}} \mathrm{SOFI}$ observations in the CDF-S generally includes some overlap of adjacent images, and, additionally, a series of lowexposure "calibration images" arranged in a pattern being offset to the deep integrations had been acquired in $J$ and $K_{\mathrm{s}}$, so that accurate photometric scalings could be measured not only between ISAAC and SOFI but also between adjacent SOFI images. To this end, we have processed the raw SOFI data in the same way as we did with the ISAAC data, employing ESO/MVM for the data reduction followed by careful examination of the image quality (Sect. 3.4). After all, 39 best-quality images in terms of seeing, noise, and background homogeneity were used in $J, H$, and $K_{\mathrm{s}}$, plus 19 shallower "calibration images" in $J$ and $K_{\mathrm{s}}$. To measure the photometric scaling between two images, at first, the image with the better seeing was smoothed with a Gaussian kernel to match the PSF of the other image with poorer seeing. Because typically the ISAAC seeing is better than the SOFI seeing (0.5" vs. $0.7^{\prime \prime}$ median), for scalings between ISAAC and SOFI images in the majority of cases the ISAAC image is smoothed to match the PSF of the SOFI image. Then, instrumental magnitudes of all isolated, high signalto-noise $(S N R>20)$ sources were measured using SExtractor's auto-scaling aperture magnitudes in double-frame mode (Bertin $\&$ Arnouts 1996) and the magnitude differences were averaged to obtain the relative photometric scaling. In this way, photometric scalings with respect to SOFI images could be measured with a typical uncertainty between 1 and $2 \%$, slightly depending on the filter. We have minimized a possible bias due to the relative nonlinearity of the two instruments as far as possible. Objects being relatively bright for ISAAC were discarded based on their peak flux corresponding to an effective flux cut at $\sim 16.5$ mag (AB). Furthermore, the photometric scaling was computed by

3 The observations have been obtained under ESO programmes 60.A9005, 164.O-0561, 66.A-0451, and 68.A-0375.

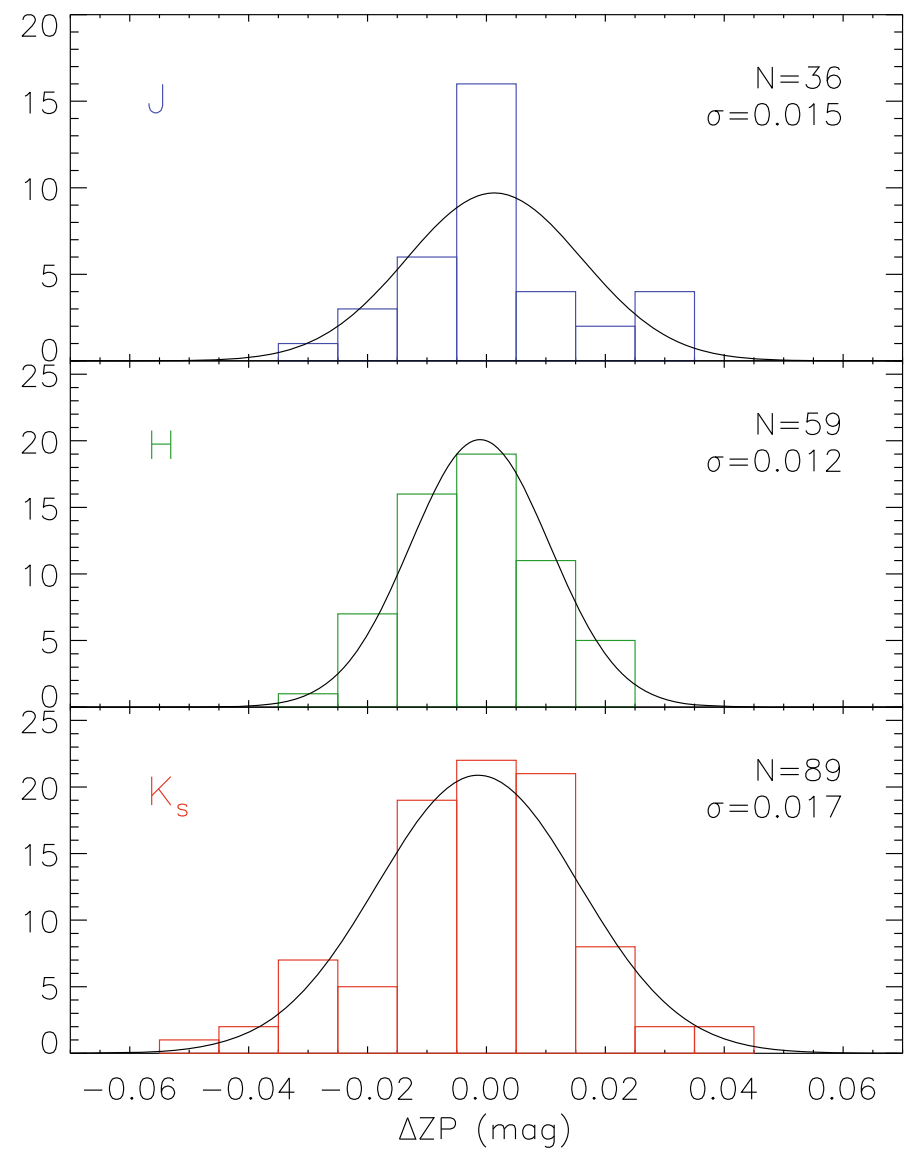

Fig. 4. Residuals of photometric zero points of $\mathrm{OB}$ images as derived from flux standards with respect to the final global photometric solution for $J, H$, and $K_{\mathrm{s}}$ (from top to bottom). For each passband, the total number of OB images having photometric zero points based on flux standards, $N$, and the RMS of the residuals about the mean, $\sigma$, is reported. Gaussian distributions with the same mean and dispersion as the actual data are superposed.

averaging over all suitable sources, i.e. typically more than 10 sources, stars and galaxies, between ca. 18 and 20 mag (AB) contribute to the resulting scaling instead of being based on a few bright stars only. For objects fainter than 17 mag, ISAAC's detector nonlinearity is not an issue (Sect. 3.5.3). As another precaution, we visually inspected the differential ISAAC-SOFI photometry but did not see any case of flux dependent bias. Therefore, we can exclude that the measured photometric scalings between ISAAC and SOFI data are biased significantly, that is by more than $0.01 \mathrm{mag}$. That followed, we have applied a global $\chi^{2}$-minimization technique (Koranyi et al. 1998), to find, for each passband, the photometric solution adjusted across the whole survey.

The residual photometric differences of the photometric ZPs as given by the flux standards with respect to the global photometric solution were examined to identify implausible ZPs. Consequently, 5 ZPs were removed, and the final photometric solution was calculated based on 155 photometric ZPs and 501 photometric scalings. This means that on average, more than one photometric ZP contributed to the final photometric solution per OB image, namely $1.4,2.5$, and 3.2 for $J, H$, and $K_{\mathrm{s}}$, respectively, thereby reducing the impact of possible errors of individual photometric ZP measurements by virtue of averaging. As an additional check, we have verified that the residuals are not correlated with observational or instrumental parameters (seeing, 


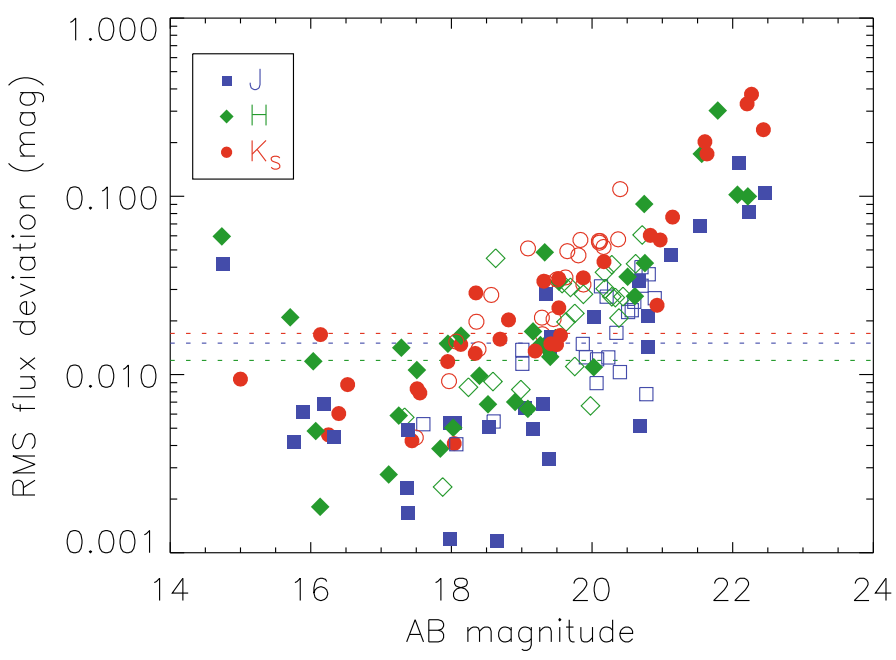

Fig. 5. Reproducibility of flux measurements on $\mathrm{OB}$ images as a function of $A B$ magnitude. Each data point corresponds to one of 56 individually selected test sources measured in one of the three filters, $J, H$, and $K_{\mathrm{s}}$, discriminated by different plot symbols, square, diamond and circle, respectively. The root-mean-square (RMS) flux deviation from the mean was computed from 3 to 30 measurements on the photometrically calibrated OB images. Solid symbols denote unresolved sources, while open symbols correspond to extended sources. For comparison the $1 \sigma$ errors of the photometric solution of the OB images (Sect. 3.5.2) are shown as horizontal dotted lines.

sky background level, DIT). The photometric residuals appear to be normally distributed, and indicate an internal photometric accuracy of better than 0.02 mag RMS from tile to tile in all three bands (Fig. 4). The individual inspection of a set of nonsaturated, isolated stars has revealed in a few cases photometric discrepancies of 3-5\% between SOFI and ISAAC images which were not attributable to photon noise but are presumably due to systematic errors left over after flat field correction.

Finally, in order to convert from the Vega to the AB system, AB corrections of $0.9603,1.426$, and $1.895 \mathrm{mag}$ for $J$, $H$, and $K_{\mathrm{s}}$, respectively, were applied. These numbers were obtained from the ESO Magnitude-to-flux-converter ${ }^{4}$ (version 1.07) which makes use of the instrumental transmission curves of the ESO Exposure Time Calculators.

\subsubsection{Photometric uncertainties}

In order to characterize the effect of detector nonlinearity on the final survey data we have performed photometric checks of individual objects on the calibrated $\mathrm{OB}$ images as a function of source properties (flux, extent), observational conditions (seeing, background brightness), and instrumental parameters (DIT). To this end five survey tiles were selected within which aperture fluxes ( 4 " diameter) of 56 isolated objects were measured in $J$, $H$, and $K_{\mathrm{s}}$ from between 3 and 30 OB images. Figure 5 summarizes the results of this study as a function of source flux. The increasing RMS flux deviation towards the bright end indicates that detector nonlinearity starts to take effect for unresolved sources at total magnitudes between 16 and 16.5 AB. The comparison with the calibrated SOFI images, which are expected to be significantly less affected by nonlinearity than the ISAAC data, allows to directly estimate the net flux bias for these objects. On average the ISAAC photometry underestimates the flux by between $0.02 \mathrm{mag}$ at $\sim 16.5 \mathrm{mag}$ and ca. 0.05 mag at

${ }_{4}$ Accessible at http://archive.eso.org/apps/mag2flux
14.7 mag AB irrespective of the pass band. The maximum flux bias of 0.07 mag was found for the $H$ flux of the brightest star in the test sample $(H=14.7)$. Note that there are actually very few objects that bright in the whole survey, namely 5 having $K_{\mathrm{s}} \leq 15$ and 23 having $K_{\mathrm{s}} \leq 16.5$, almost all of them being stars with the exception of two bright galaxies of $K_{\mathrm{s}}=16.1$ and 16.5. For the bulk of objects having total fluxes of 17 mag $\mathrm{AB}$ and fainter, no indication for nonlinearity effects has been found. In fact, the overall trend of the flux uncertainty, which resolved and unresolved objects seem to follow in the same way, can be explained purely in terms of statistical errors (see also Sect. 4.2).

Figure 5 also indicates the errors of the photometric solution to illustrate the level of systematic photometric errors in proportion to the statistical errors. For instance, between ca. 16 and $18 \mathrm{mag}, H$ and $K_{\mathrm{s}}$ photometry is typically limited by the systematic uncertainties intrinsic to the photometric solution, or, photometry fainter than $J \sim 20$ becomes dominated by statistical errors. Remember that the data points in Fig. 5 refer to the $1 \sigma$ fluctuations of photometric measurements on OB images, whereas the final co-added survey images are expected to have reduced photometric errors, typically by a factor of between 2 and 2.5 .

Exceptionally high sky background brightness in combination with a comparatively large detector integration time (DIT) has resulted in a few OBs in $H$ and $K_{\mathrm{s}}$ for which the detector was not operated in the linear regime. In fact, the most extreme cases are 8 out of $294 \mathrm{OBs}$ in $H$ and $K_{\mathrm{s}}$ for which the sky background signal induces a nominal nonlinearity of between 2 and $3 \%$. To compensate for this effect which is effectively resulting in a loss of sensitivity, or a lowered photometric $\mathrm{ZP}$, the respective OBs were not directly anchored to ZPs but their photometric calibration was inferred from relative photometric scalings with respect to the other OBs of the same tile and filter (see Sect. 3.5.2). Finally, we have verified that the photometric measurements on the calibrated "nonlinear" OBs are indistinguishable from the other data.

To estimate the overall error, we linearly add up the individual contributions, that is the internal accuracy of the photometric solution $(\leq 0.017 \mathrm{mag})$, the residual flat field error $(0.025 \mathrm{mag}$, $1 \sigma)$, and the possible nonlinearity bias $(\lessgtr 0.01 \mathrm{mag})$ for objects not brighter than 17 mag $(\mathrm{AB})$, resulting in the total photometric error of up to $0.05 \mathrm{mag}(1 \sigma)$.

\subsection{Final image co-addition}

The co-addition of the 378 OB images into 78 survey tile images marks the final step of the image data reduction process (cf. Fig. 2). There is no resampling involved in this step because the $\mathrm{OB}$ images are already defined on the final astrometric grid.

At first, OB images were rescaled to the fiducial $\mathrm{ZP}$ of $26.0 \mathrm{mag}(\mathrm{AB})$, which in fact is quite close to the typical value for all three bands (see Fig. 3). Relative weights between OB images contributing to the same tile were chosen to be proportional to the inverse variance of the flux integrated within the circular aperture that maximizes the total limiting magnitude for point sources (c.f. Sect. 4.2). The weight map associated with each $\mathrm{OB}$ image was employed to consistently accounted for the positional dependence of the variance. By comparison with different weighting schemes, we have experimentally verified that the adopted scheme indeed optimizes the resulting image depth for point sources as intended. The associated final weight maps are inverse variance maps and were rescaled to the actual, empirically determined pixel-to-pixel variance of the sky background. For the final survey images the gain factor, $G_{0}$, that is the number of detector electrons per data unit, is reported in Table 2, last 


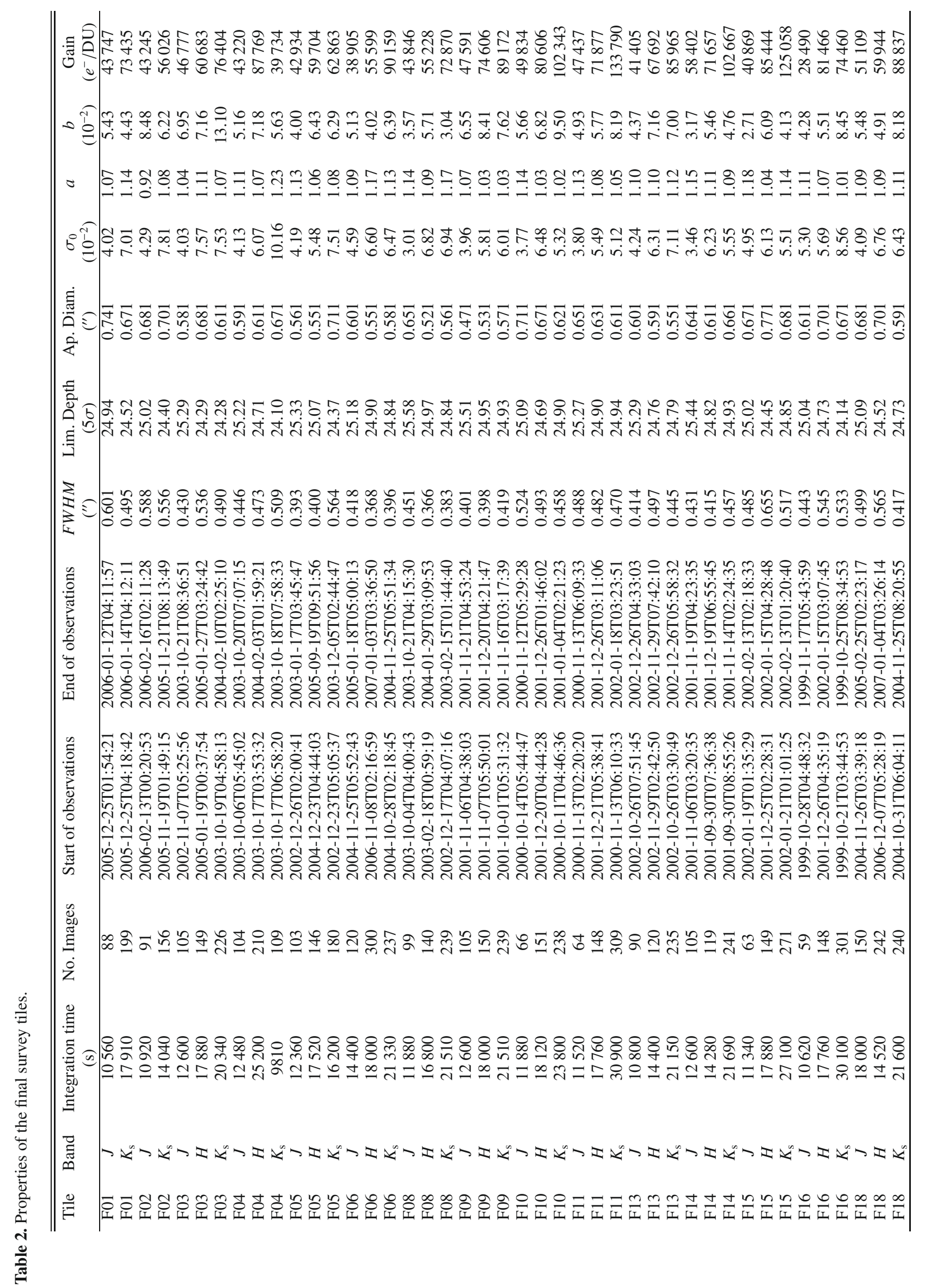




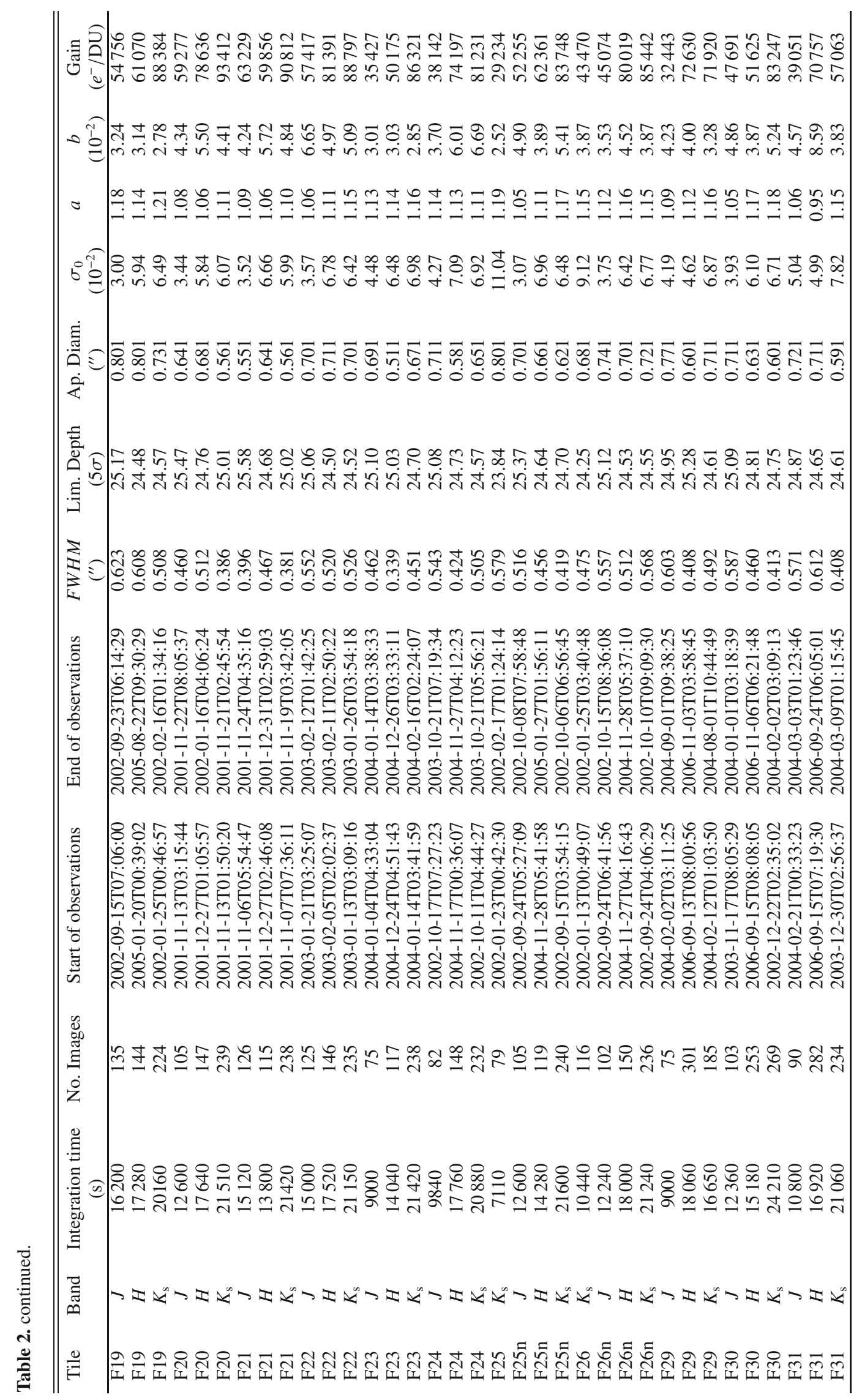




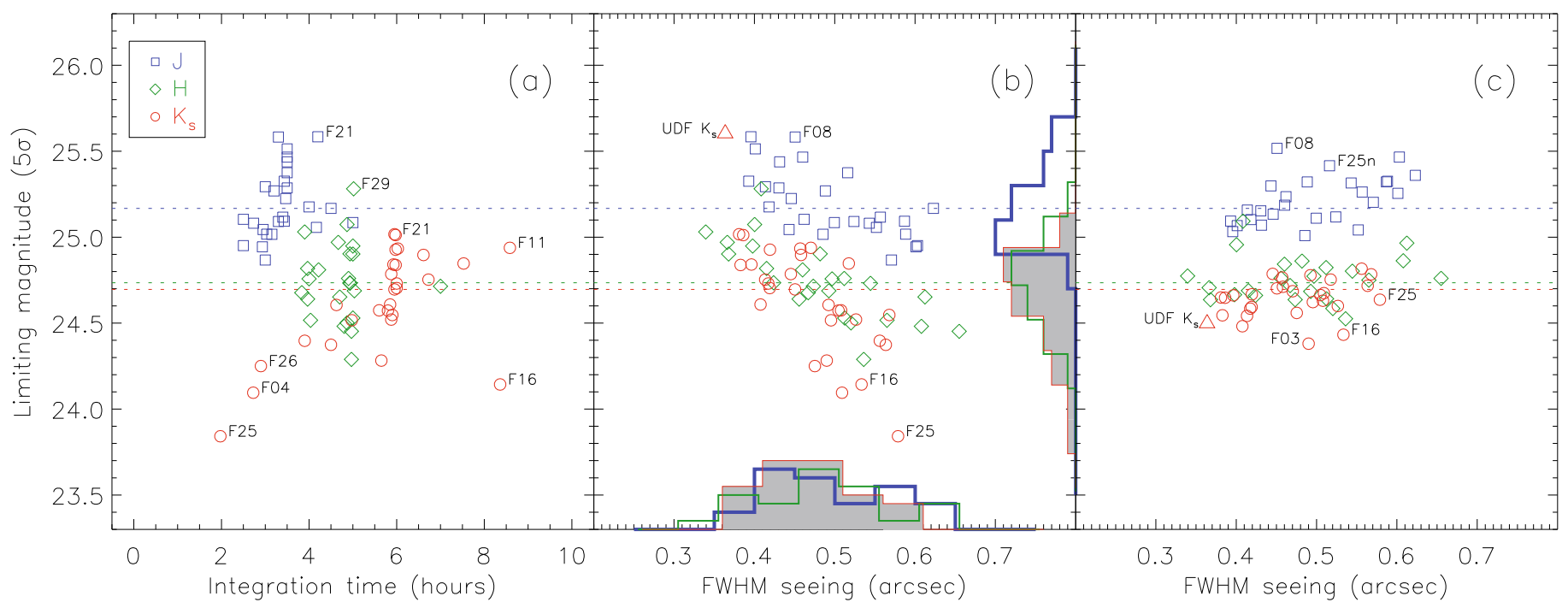

Fig. 6. Limiting magnitude ( $5 \sigma$, total for point sources) of the final survey tiles as a function of a) total integration time, and, b) PSF size ("seeing"). Panel c) shows the limiting magnitude corrected for the variation of the instrumental zero point and scaled by exposure time and seeing so that the dependencies formally cancel out (see text). The survey tiles are marked with square, diamond and circle symbols as indicated in the legend. The triangle symbol represents the UDF/ISAAC $K_{\mathrm{s}}$ image. Dotted lines denote the median depth of the GOODS/ISAAC tiles. Histograms of seeing and limiting magnitude are marked by thick line (blue), moderately thick line (green), and normal line (red) plus grey shade for $J, H$, and $K_{\mathrm{s}}$, respectively.

column. As $G_{0}$ refers to the median pixel, the effective gain at pixel $j$ can be obtained by scaling with the relative (i.e. mediannormalized) weight, $w_{j}$, according to $G_{j}=G_{0} w_{j}$.

In addition to the individual image tiles, the data release also includes mosaics of the co-adjoined tiles as single FITS files in $J, H$, and $K_{\mathrm{s}}$ bands, as well as the corresponding weight maps. The net area is $172.5,159.6$ and $173.1 \operatorname{arcmin}^{2}$ in $J, H$, and $K_{\mathrm{s}}$, respectively. The WCS information and accuracy of the individual tiles is preserved in these mosaics. A uniform ZP of 26.0 mag can be used (e.g. with SExtractor) across the entire field, however, it is important to note that the PSF varies from tile to tile within each mosaic. In the absence of proper aperture corrections or PSF matching procedures, this would lead to biases when creating multi-color catalogs. A possible procedure for coping with the PSF variations without resorting to image convolution is outlined in Sect. 5.2.

\section{Final survey images}

\subsection{General properties}

In the following, we summarize and discuss the properties of the final survey images as presented in Table 2. The recorded parameters are: total integration time, total number of raw images which were combined to form the final product, the period during which observations where conducted, the $F W H M$ of the PSF ("seeing") in arcseconds, the $5 \sigma$ limiting magnitude correct to the total flux assuming a point source profile ("image depth"), the aperture diameter to which the depth refers, the 3 parameters of the noise model $\sigma_{0}, a, b$, and the effective gain.

Summing the actual exposure time from the data that were combined in the final stacks yields a total amount of integration time of $359.9 \mathrm{~h}$. Regarding the distribution of integration time that went into the final images, one notices many tiles standing out with respect to the nominal values (Fig. 6a). This is because OBs which were rejected in the course of the final quality control process could not be re-scheduled for observations leading to reduced integration time for those tiles with respect to the nominal values for the survey. For instance, in the most extreme case $\left(\mathrm{F} 04 K_{\mathrm{S}}\right)$, three out of six OBs were rejected - two OBs for high noise, one for bad seeing. The two "supplementary" tiles, F25 and F26, are an exception in the sense that they have just a fractional amount of observation time by design. On the other side, there are tiles with integration times in excess of the nominal values, most notably F11 and F16 in $K_{\mathrm{s}}$, which is primarily due to the fact that data from several observing programs have been combined.

The PSF of the final images, quantified by its FWHM using the same methodology as detailed in Sect. 3.4, varies between $0.34^{\prime \prime}(\mathrm{F} 23 H)$ and $0.65^{\prime \prime}(\mathrm{F} 15 H)$ with a median value of $0.48^{\prime \prime}$ and appears to be independent of the pass band (Fig. 6b). We have checked that the final FWHM does not show any correlation with the number of raw images or the total integration time, which indicates that the image reduction process does not degrade the PSF quality. Generally, we have found a low level of PSF anisotropy. SExtractor-measured ellipticities are typically smaller then 0.05 - sometimes even clearly below 0.03 .

Based on the analysis of the curve of growth of unsaturated isolated stars using circular apertures between $0.7^{\prime \prime}$ and $10^{\prime \prime} \mathrm{di}-$ ameter, we have quantified the aperture corrections for point source photometry, $\delta m_{\mathrm{ap}}$, for each tile (Table 3). $10^{\prime \prime}$ has been adopted as the reference aperture for the practical reason, that this turns out to be the upper limit out to which the correction can be traced in case of most favorable conditions, i.e., if sufficiently bright stars are present in the image. In other cases when appropriate stars are lacking - a power-law extrapolation of the curve of growth had to be applied to obtain the data for the largest apertures. For all images the curve of growth falls significantly below the $1 \%$ level at the end which justifies to consider the $10^{\prime \prime}$ flux as the total flux, and to infer the total magnitude, $m_{\mathrm{tot}}$, from the aperture magnitude, $m_{\mathrm{ap}}$ by means of $m_{\mathrm{tot}}=m_{\mathrm{ap}}-\delta m_{\mathrm{ap}}$ as is done throughout the rest of the work. 


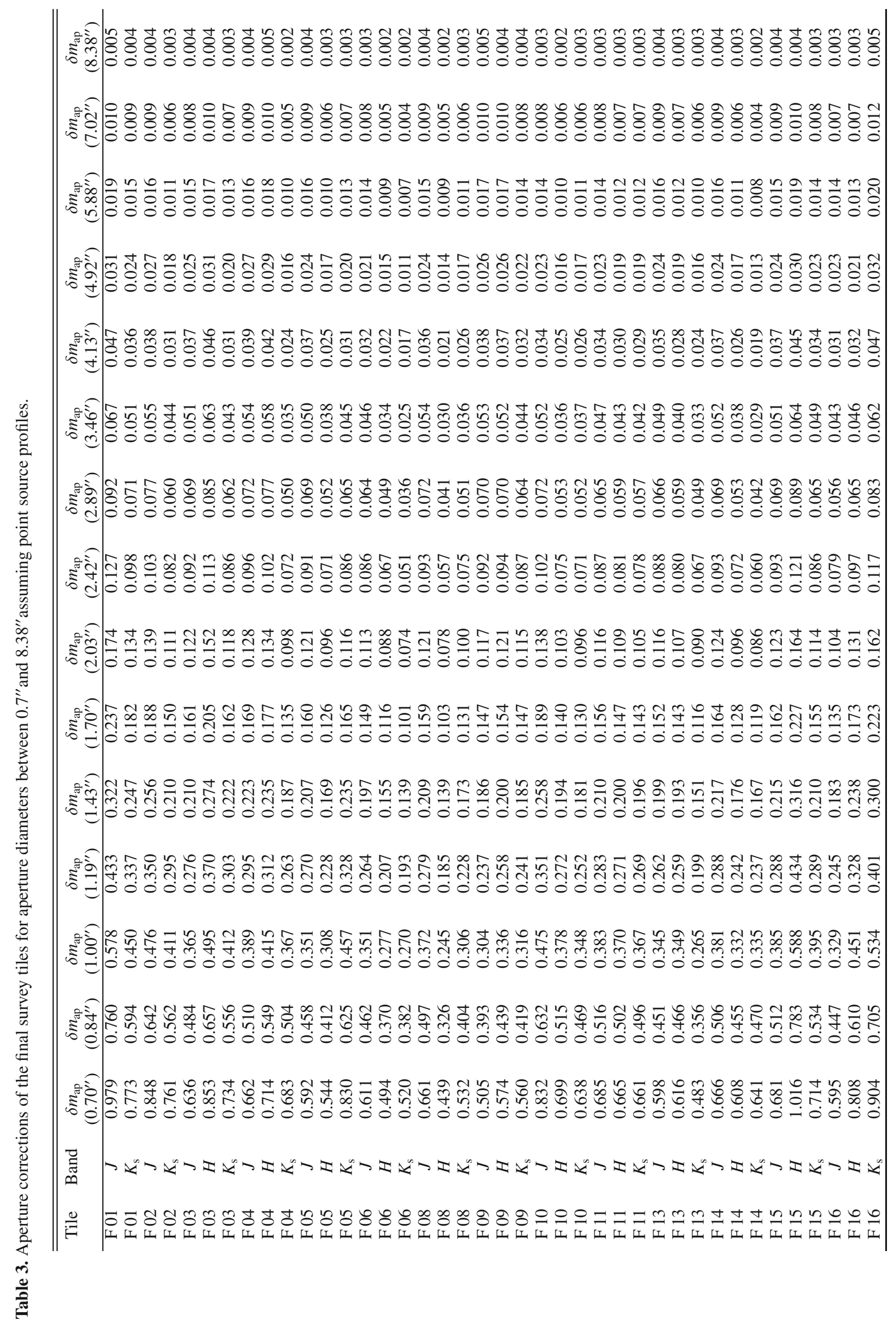




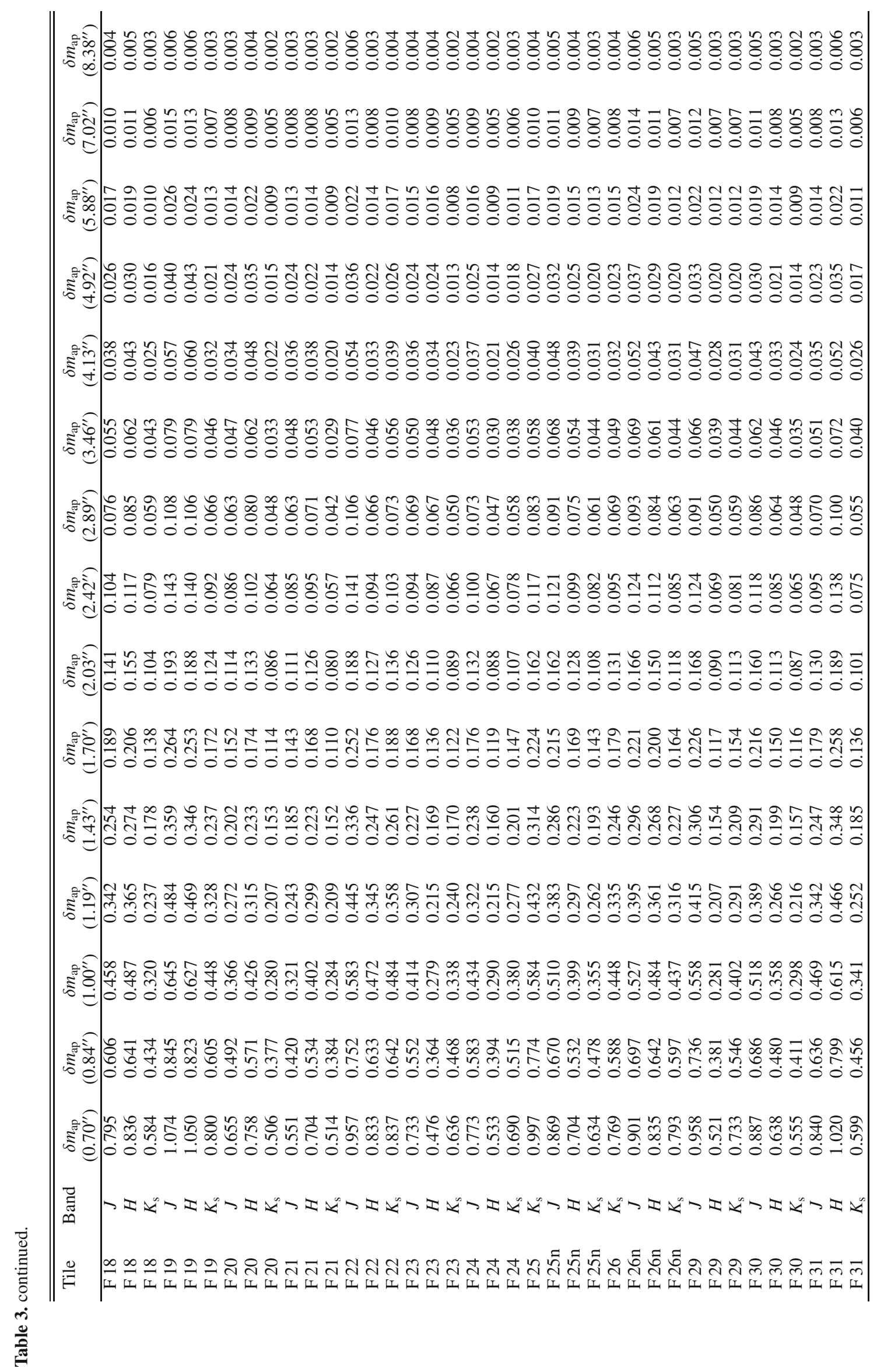




\subsection{Sky background and limiting magnitude}

First, we have evaluated the sky background noise using the same methodology as detailed in Sect. 3.4. That is, for each image, we have obtained a model for the sky noise according to Eq. (1) in terms of the 3 parameters $\sigma_{0}, a$, and $b$. Below, we will also employ these numbers to estimate flux errors (Sect. 5.1). Then, the total magnitude for point sources being associated with the sky background fluctuation was computed taking into account the aperture correction, $\delta m_{\mathrm{ap}}$, and the photometric ZP,

$m_{\mathrm{lim}}=-2.5 \log \sigma_{\mathrm{sky}}-\delta m_{\mathrm{ap}}+\mathrm{ZP}$,

and its maximum with respect to the aperture diameter was identified as the point source limiting magnitude of the given image at $1 \sigma$ significance level. As customary, we refer to the $1.747 \mathrm{mag}$ brighter $5 \sigma$ limiting magnitude in the following. The variation across the image is given by the weight map, $w_{j}$, according to $m_{\lim }=m_{\lim }^{(0)}+1.25 \log w_{j}$, whereas $m_{\lim }^{(0)}$ refers to the median pixel by convention. The median limiting magnitude and the diameter of the corresponding circular aperture are listed in Table 2.

The primary dependencies of the limiting magnitude of the final images on total integration time and atmospheric seeing are shown in Fig. 6a,b. However, there are considerable deviations for individual tiles. $\mathrm{F} 16 K_{\mathrm{s}}$, for instance, turns out to be much shallower than expected based on its substantial total integration time which is a consequence of the joined effect of worsethan-average seeing and the lower instrumental sensitivity in this period (see also Fig. 3). The median of the depth of the final images is 25.2 for $J$, and 24.7 both for $H$ and $K_{\mathrm{s}}$, which is in good agreement with the original survey goals (cf. Sect. 2). Scaling the limiting magnitude to correct for the primary determining factors, namely integration time $\left(t_{\text {intg }}\right), F W H M$, and the effective variation of the instrumental zero point for this image, $\Delta \mathrm{ZP}$, according to

$m_{\text {lim }}-1.25 \log t_{\text {intg }}+2.5 \log F W H M-\Delta \mathrm{ZP}$,

leaves a variation about the mean of between 0.11 and 0.14 mag RMS (Fig. 6c). This is caused by sky brightness fluctuations. There is the simple effect that the sky noise is higher when the sky is brighter-i.e., shot noise variations due to the fact that the sky is brighter on some nights than on others. Moreover, shortterm fluctuations in the atmospheric sky brightness on a time scale similar to the integration time of an OB ultimately limit the accuracy with which the sky background can be subtracted during image data reduction.

To quantify the depth of the survey as a whole, the effective area, that is the cumulative area distribution as a function of limiting magnitude, has been computed (Fig. 7). As the overlap of tiles is taken into account, the effective area corresponds with the final survey mosaics. One reads off that within $90 \%$ of the nominal survey area the limiting magnitude is 24.8, 24.3, and 24.1, within $75 \%$ it is $25.0,24.5$, and 24.4 , and within $50 \%$ it is 25.1 , 24.7 , and 24.7 for $J, H$, and $K_{\mathrm{s}}$, respectively.

\subsection{Validation of the photometric calibration}

We have conducted several checks to verify the consistency of the photometric calibration of the final survey images starting with an internal check based on multiple detections, followed by the comparison with an independently reduced and calibrated subset of the same raw data, and, finally, by comparison with external NIR photometric data having been published in the literature.

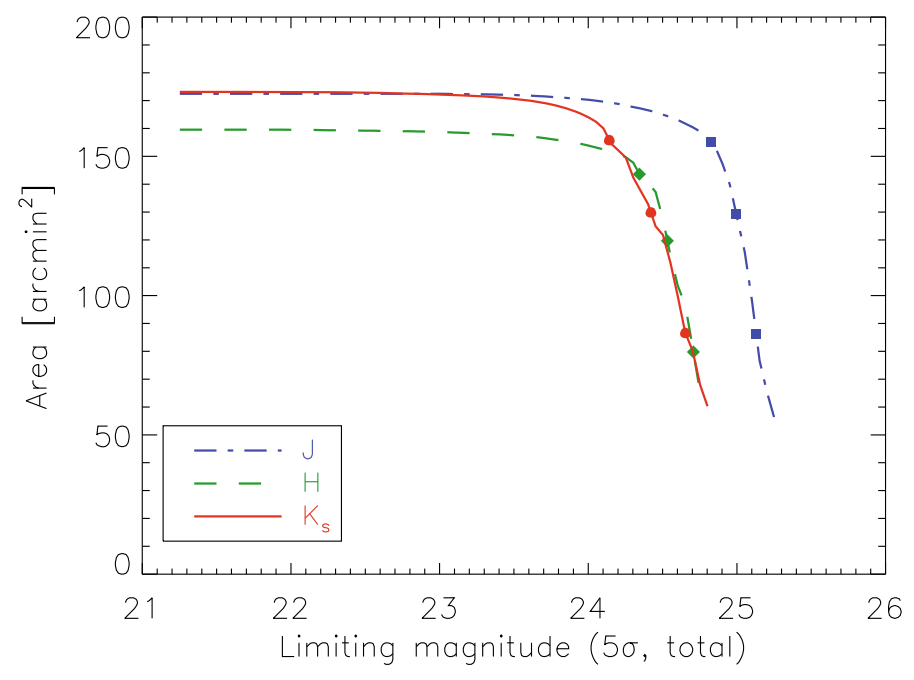

Fig. 7. Effective survey area vs. limiting magnitude for $J, H$, and $K_{\mathrm{s}}$ bands. Plot symbols mark $90 \%, 75 \%$, and $50 \%$ of the total survey area for which the limiting magnitudes are given in the text.

At first, we have analyzed sources having multiple detections to verify the internal consistency of the photometric calibration of the final survey images. To this end, photometric differences of multiple detections of non-blended sources were inspected as shown in Fig. 8 a-d. Total magnitudes have been used in order to minimize any possible effect due to seeing variations between different tiles. We do not see any systematic discrepancies in this test, rather, at large, we found that the scatter appears to be compatible with the formal error bars. For $J$ and $H$, the sources are located in the overlapping region of adjacent survey tiles, whereas for $K_{\mathrm{s}}$ band, we took advantage of the "supplementary" survey fields F25 and F26 which mark a part of the survey area that is covered twice. Consequently, in case of $J$ and $H$, the amount of scatter about the zero line is enhanced because the sources lie towards the border of the image tiles where the exposure time of the jitter observations is effectively reduced with respect to the central part of the image, and, in addition, where possible flatfielding errors may be expected to increase. Regarding the two fields in $K_{\mathrm{s}}$, the larger scatter of F25 over F26 is due to the fact that $\mathrm{F} 25 K_{\mathrm{S}}$ is about $0.4 \mathrm{mag}$ shallower than F26 $K_{\mathrm{s}}$ (cf. Sect. 4.2). Generally, the overall scatter of measured fluxes with respect to true fluxes is expected to be smaller than the scatter shown here.

Next, we have investigated whether the adopted strategy of data reduction and calibration procedures affect the final photometric results. To this end, we have used the $J$ and $K_{\text {s }}$ photometric data from Saracco et al. (2001) which is based on the same set of raw data that went into the production of tile F16 of the GOODS/ISAAC survey and allows to carry out a comparison that is unaffected by varying observing conditions or different instrumental characteristics. Here, total magnitudes were used in order to be compatible with the combination of corrected $2^{\prime \prime}$ apertures and BEST apertures used by Saracco et al. (2001). The results, displayed in Fig. $8 \mathrm{e}-\mathrm{f}$, show that both data sets are photometrically compatible, except for very few outliers.

Furthermore, we have checked our survey's photometry against external NIR catalogs of the CDF-S region which have been obtained and calibrated independently from the GOODS/ISAAC data (Fig. 9). To this end, common sources were identified using a maximum pairing distance of $1^{\prime \prime}$ from which only isolated i.e. non-blended sources were selected. For the comparison with the Two Micron All Sky Survey (2MASS, 

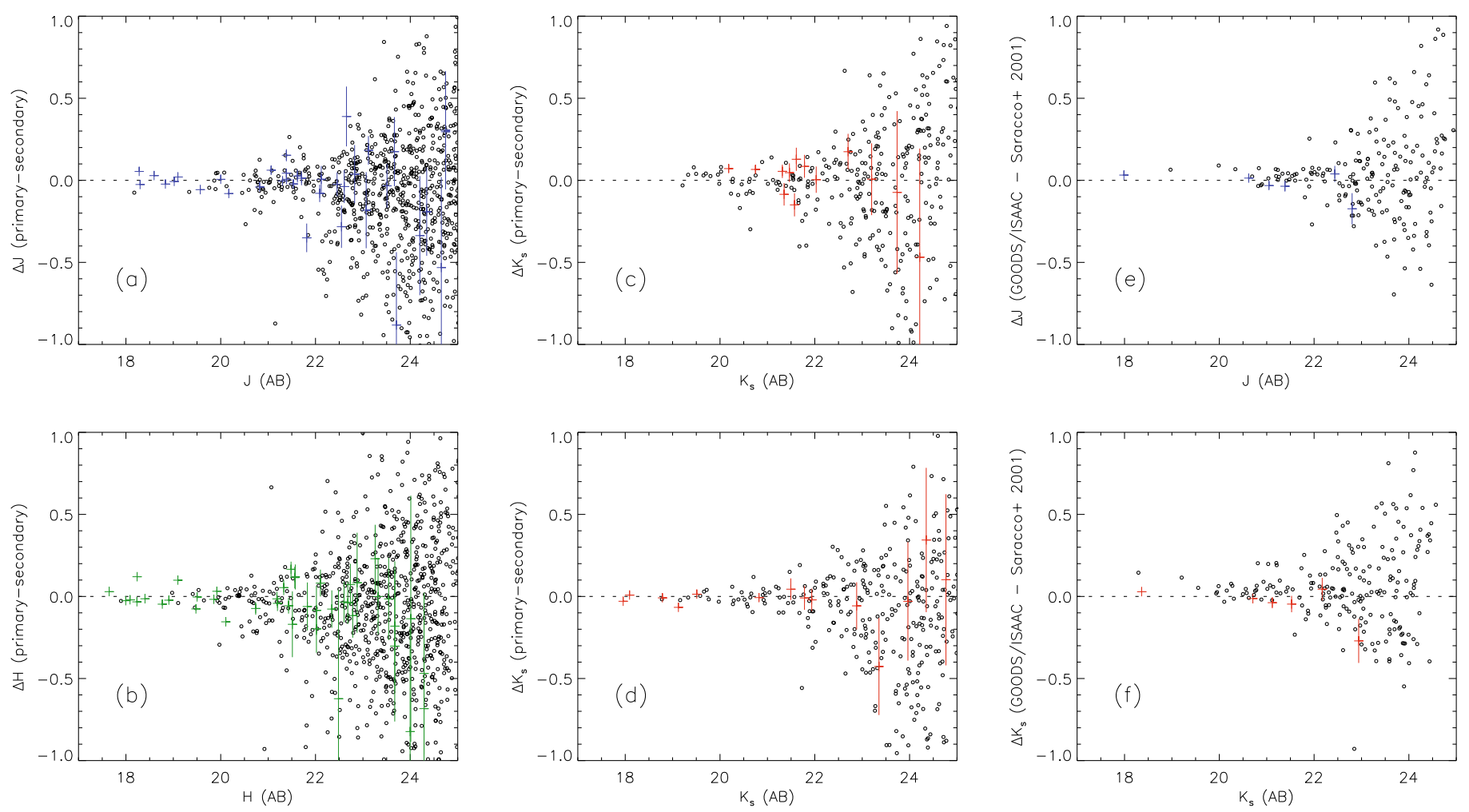

Fig. 8. Photometric comparison of primary and secondary detections in overlapping regions in $J$ and $H \mathbf{a}$ ), b), similarly, based on the "supplementary" survey fields F25 and F26 in $K_{\mathrm{S}}$ band c), d), and, with respect to the photometric catalog by Saracco et al. (2001) in $J$ and $K_{\mathrm{s}}$ e), f). Point-like sources are indicated by cross symbols with $1 \sigma$ vertical error bars for the magnitude difference, whereas extended sources correspond to the circle symbols.

Skrutskie et al. 2006), we also made use of the SOFI data set which had been used before to establish the survey's global photometric solution in order to extend the magnitude range for the comparison towards the bright end. In this case, just point sources were considered. Fluxes were measured in apertures of varying size (while maximizing the signal-to-noise ratio) and were corrected for aperture losses to a common aperture diameter of $10^{\prime \prime}$ which is virtually equivalent to a total magnitude given the typical PSF. We applied the transformation between the 2MASS and LCO photometric system as determined by Carpenter (2001), and used $J-J_{2 \text { MASS }}=0.022$, $H-H_{2 \mathrm{MASS}}=0.012$, and $K_{\mathrm{s}}-K_{\mathrm{s}, 2 \mathrm{MASS}}=0.015$, whereas neglecting any color terms. The trend of measured source fluxes being apparently fainter than 2MASS fluxes at magnitudes faintward of $17.5 \mathrm{AB}$ is the result of a Malmquist-like selection effect and is also present in the comparison with other data sets that are significantly shallower than GOODS/ISAAC. Taking this effect into account, the data exhibits no evidence for a bias in the photometric calibration with respect to 2MASS. The scatter of the data is basically consistent with the formal flux errors which are clearly dominated by 2MASS. It is worth noting that consistent aperture corrections turned out to be crucial in this test. In fact, a lack thereof results in a significant bias at the $\gtrsim 0.1 \mathrm{mag}$ level which is visible despite the significant scatter.

As we did not apply corrections for nonlinearity in the course of data reduction, neither for ISAAC nor for SOFI data, differential nonlinearity may in principle affect any photometric comparison. To address this issue we followed the same procedure as described in Sect. 3.5.2 and discarded relatively bright, potentially nonlinear objects from the photometric analysis (independently in the ISAAC as well as in the SOFI data), thereby reducing nonlinearity effects in our data to nominally $\lesssim 0.01 \mathrm{mag}$ and, thus, making them basically negligible with respect to the other sources of error. In terms of 2MASS the reference sources used here are relatively faint, which means that the photometric uncertainties are dominated by background noise and nonlinearity is expected to be insignificant.

For the following comparisons with catalogs which are comparatively deeper than 2MASS, we used SExtractor's autoscaling magnitudes for formal consistency with the type of magnitudes published. However, photometric apertures were not matched for lack of detailed information about the apertures and the associated flux corrections for the reference data sets. Thus, a systematic effect depending on the data set is to be expected. The comparison with the EIS-DEEP data set in $J H K_{\mathrm{S}}$ is based on the most recent processing done by Olsen et al. (2006). The differences of the $\mathrm{AB}$ corrections adopted in their and in our work are corrected for. It turns out that magnitudes from GOODS/ISAAC and EIS-DEEP are consistent modulo a constant offset of $\approx 0.1 \mathrm{mag}$ for $J$ and $H$, and certainly $<0.1 \mathrm{mag}$ for $K_{\mathrm{s}}$. The apparent scatter is larger than what the individual flux error estimates suggest - a finding that applies to this and the following comparisons as well. The magnitude differences with respect to the $H$-band observations of the CDF-S by Moy et al. (2003) are noticeably more dispersed while not exhibiting any systematic offset. The comparison with the Las Campanas Infrared survey (LCIRS, Chen et al. 2002) features a slight trend with magnitude in such a way that bright sources $(H \lesssim 18)$ appear brighter in GOODS/ISAAC while faint sources appear to be unbiased or even slightly fainter than in the LCIRS. The 
J. Retzlaff et al.: The Great Observatories Origins Deep Survey
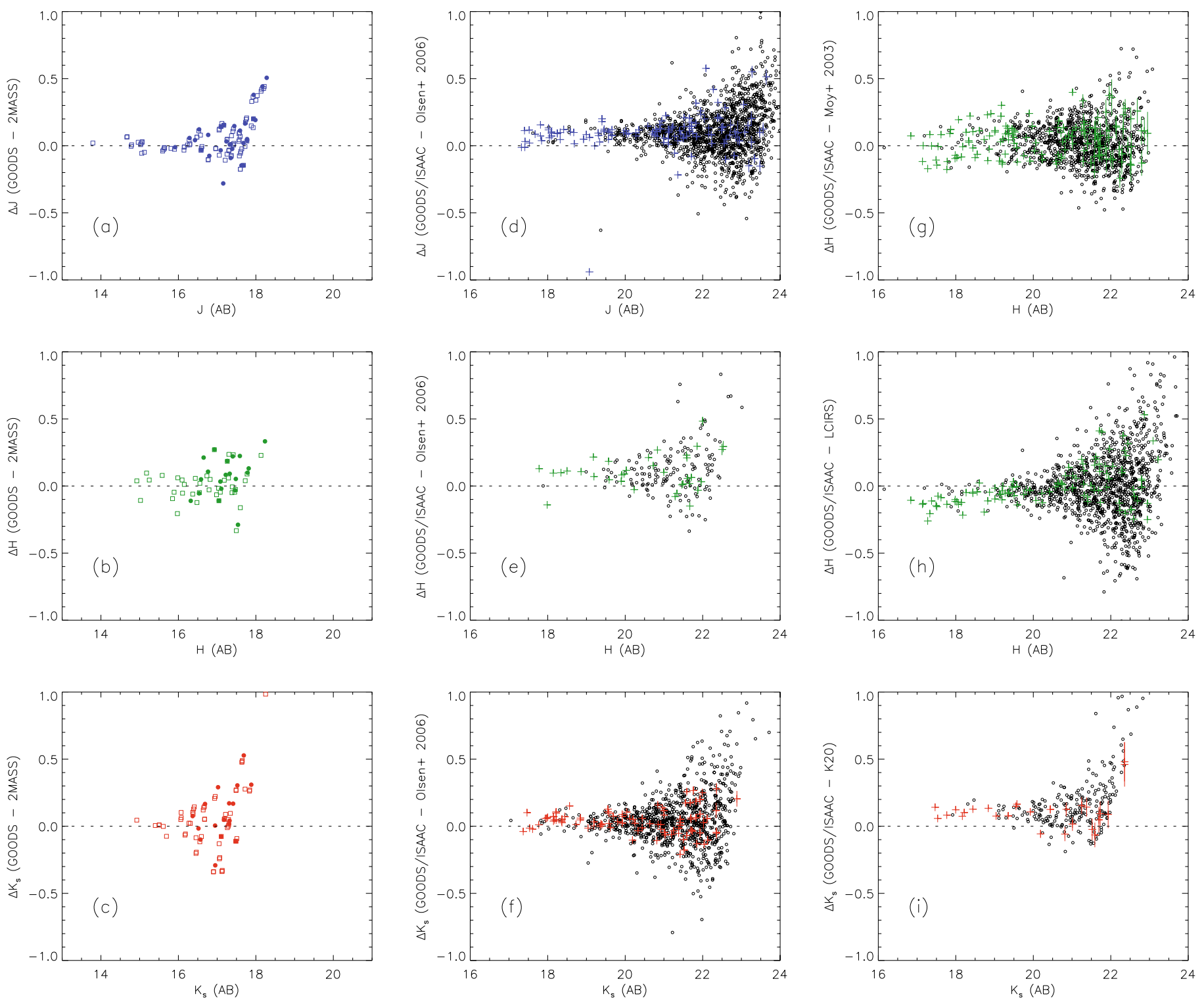

Fig. 9. Photometric comparison with respect to public NIR catalogs of the CDF-S region. Each panel corresponds to one reference data set in one filter as indicated in the label, namely, a)-c) Two Micron All Sky Survey (Skrutskie et al. 2006), d)-f) ESO imaging survey (Olsen et al. 2006), g) $\mathrm{H}$-band observations of the CDF-S by Moy et al. (2003), h) Las Campanas Infrared survey (Chen et al. 2002), and, i) K20 survey (Cimatti et al. 2002). The differences of magnitudes of sources as measured on the GOODS/ISAAC images and as given in the respective reference catalogs are displayed as a function of the measured GOODS/ISAAC magnitude in the AB system. In case of 2MASS, dot symbols correspond to measurements performed directly on the calibrated ISAAC survey fields while square symbols denote measurements on the "auxiliary" SOFI images which were used to homogenize the photometric solution (see text). For the comparison with the other - much deeper - catalogs crosses and circles correspond to point-like and extended sources, respectively, with 1-sigma error estimates on the magnitude difference being indicated for point-sources if exceeding the size of the plot symbol.

magnitudes of the K20 galaxy survey (Cimatti et al. 2002) appear to be brighter by $\approx 0.1 \mathrm{mag}$ than the respective GOODS/ISAAC magnitude.

As a result, we do not find that photometric differences with respect to various independent data sets exhibit any coherent trend being attributable to the GOODS/ISAAC survey. Rather, it seems to be plausible to suppose that significant photometric differences are mostly due to the photometric calibration, the exact definition of photometric apertures, the effect of seeing variations and, possibly, the lack of correction thereof of individual reference data sets. A mismatch of photometric apertures can make up a systematic difference of $\sim 0.1 \mathrm{mag}$. For example, correcting the GOODS/ISAAC magnitudes for aperture losses by using total magnitudes completely eliminates the systematic offset with respect to K20. After all, the excellent photometric agreement with 2MASS in $J$ and $K_{\text {s }}$ provides strong evidence that the global photometric error of the GOODS/ISAAC survey tiles is significantly below the 0.1 mag level.

\section{Source catalogs}

\subsection{Source detection and photometry}

We use SExtractor (Bertin \& Arnouts 1996) for source detection adopting a spatial filtering with a 2-dimensional Gaussian of 2.5 pixels $F W H M$ to match with the PSF of the best seeing images. A detection threshold of 1.35 times the local background RMS has been applied with a minimum detection area of 

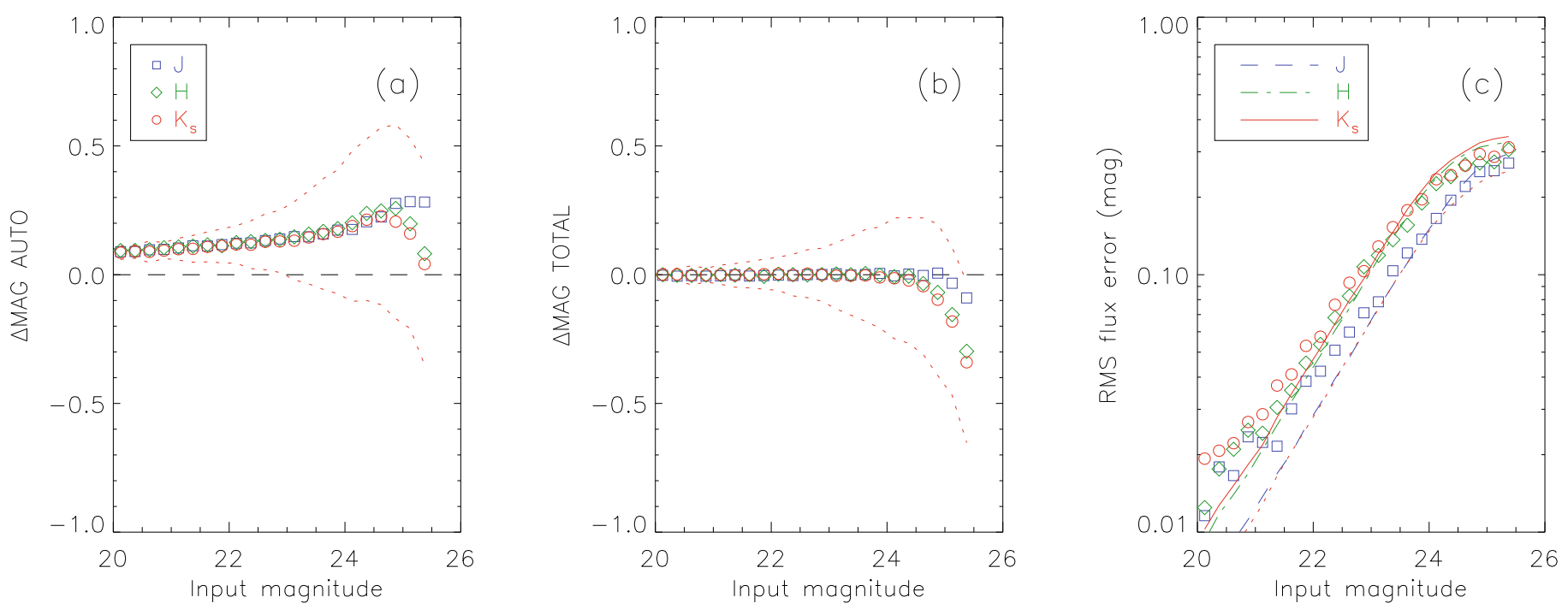

Fig. 10. Performance of photometric estimators as inferred from image simulations. Ratio of recovered flux and input flux (magnitude scale) for a) Kron-like apertures (MAG AUTO), and b) total, i.e. aperture-corrected, flux (MAG TOTAL). The binned average is plotted for $J$, $H$, and $K_{\mathrm{s}}$ with square, diamond, and circle symbols, respectively. The dotted line indicates the RMS scatter per bin for $K_{\mathrm{s}}$. Panel c) contrasts the actual flux errors computed in the RMS sense per bin (square, diamond, and circle symbols) with the flux error estimate according to Eq. (4) (dashed, dash-dotted, and continuous line). The standard SExtractor flux error estimate for $K_{\mathrm{s}}$ is shown as dotted line.

one pixel. This choice corresponds to a $5 \sigma$ significance once the spatial filtering is taken into account. The choice of detection threshold and minimum detection area has been gauged based on the analysis of catalog completeness and contamination for a grid of detection parameters (cf. Sect. 6.1). For the given spatial filtering, we have tested in particular if a minimum detection area of more than one pixel is favorable. However, it turned out that this does not increase the catalog completeness at a given contamination level, neither for point-sources nor for extended sources. The background map has been estimated in square cells of 200 pixels on a side using a $5 \times 5$ cells median filtering. For photometry a local background annulus in square shape with 20 pixels width was used.

We have adopted the prescription of Labbé et al. (2003, Sect. 5.2) to define the aperture-corrected total flux and a flux from which colors are computed based on the $K_{\mathrm{s}}$ band apertures. For isolated sources the total flux measurement is based on SExtractor's auto-scaling elliptical apertures (MAG AUTO) inspired by Kron (1980) corrected for the flux loss due to the finite aperture size. For the parameters which control the size of the auto-scaling apertures, we have used the default values, that is $k=2.5$ for the scaling parameter and $R_{\min }=3.5$ for the lower bound of the aperture size (in isophotal units). To apply the image-specific aperture corrections as given in Sect. 4.1, we have converted the elliptical aperture area into the corresponding circular aperture's diameter. In order to minimize the mutual flux contamination for blended sources, in this case the total flux measurement is based on a reduced circular aperture whose area is half the isophotal area. To prevent too small and too large apertures that are more error-prone, the aperture diameter is bound by 0.7 " and 2.0", respectively. The "color flux" is isophotal (as defined in $K_{\mathrm{s}}$ ) if the source is isolated and if the isophotal area ranges between the size of a circular aperture of $0.7^{\prime \prime}$ and $2.0^{\prime \prime}$ diameter. For sources whose isophotal areas exceed these limits, the aperture flux at $0.7^{\prime \prime}$ or $2.0^{\prime \prime}$ is assigned instead. For blended sources, the color flux is equally defined as for the total flux, based on the flux measured within a "reduced" circular aperture.

We computed flux errors taking into account the background fluctuations, both for the source aperture and for the background region, and the contribution due to the discrete nature of the detector electrons. To this end, the noise model established in Sect. 4.2 was evaluated at the spatial scales corresponding to the aperture size, $A$, and the size of the background region, $B$, respectively. With the discreteness noise which has been estimated based on the local gain, $G$, the flux error is given by

$\Delta f=\sqrt{\sigma_{\text {sky }}^{2}(A)+\sigma_{\text {sky }}^{2}(B)+\frac{f}{G}}$.

We investigated the performance of flux and flux error measurements by means of simulated images, that is actual survey tiles into which stellar sources of known flux had been embedded (cf. Sect. 6.1.2). Figure 10a shows that SExtractor's MAG AUTO measurement suffers a systematic flux loss of $\approx 0.1$ mag at 20 mag that increases towards fainter fluxes, amounting $\approx 0.2 \mathrm{mag}$ (at $24 \mathrm{mag}$ ) for isolated sources throughout all bands. The apparent reduction of the average flux loss faintward of $24.5 \mathrm{mag}$ is due to the selection effect which renders the distribution to be significantly skewed towards larger measured flux (Malmquist bias), and since this bias is determined by the signalto-noise ratio, the effect for the $J$ band is less pronounced than for $H$ and $K_{\mathrm{s}}$. Figure $10 \mathrm{~b}$ demonstrates that the measured total flux, denoted MAG TOTAL, is unbiased.

Figure 10c illustrates that the flux error computed according to Eq. (4) is able to represent the true flux errors quite accurately over a wide range of fluxes. In contrast, SExtractor's standard flux error (MAGERR AUTO) systematically underestimates the true flux errors by at least a factor of $\sim 1.5$ (in mag) for all fluxes. This discrepancy underlines that it is essential to take into account the actual local background fluctuations for realistic flux error computations instead of simply scaling the pixel-topixel variance. In detail, the estimated flux error is in fairly good agreement faintward of $\approx 22 \mathrm{mag}$. The apparent overestimation of the flux errors for the faintest fluxes ( $\gtrsim 24 \mathrm{mag}$, especially for $H$ and $K_{\mathrm{s}}$ ) is probably an artefact due to the strong selection effect in this regime. Towards bright fluxes, errors are increasingly underestimated, for instance, by about a factor of two at $K_{\mathrm{s}} \approx 20$, because systematic effects that are beyond the simple statistical error model, such as source blending, start to become dominant. 


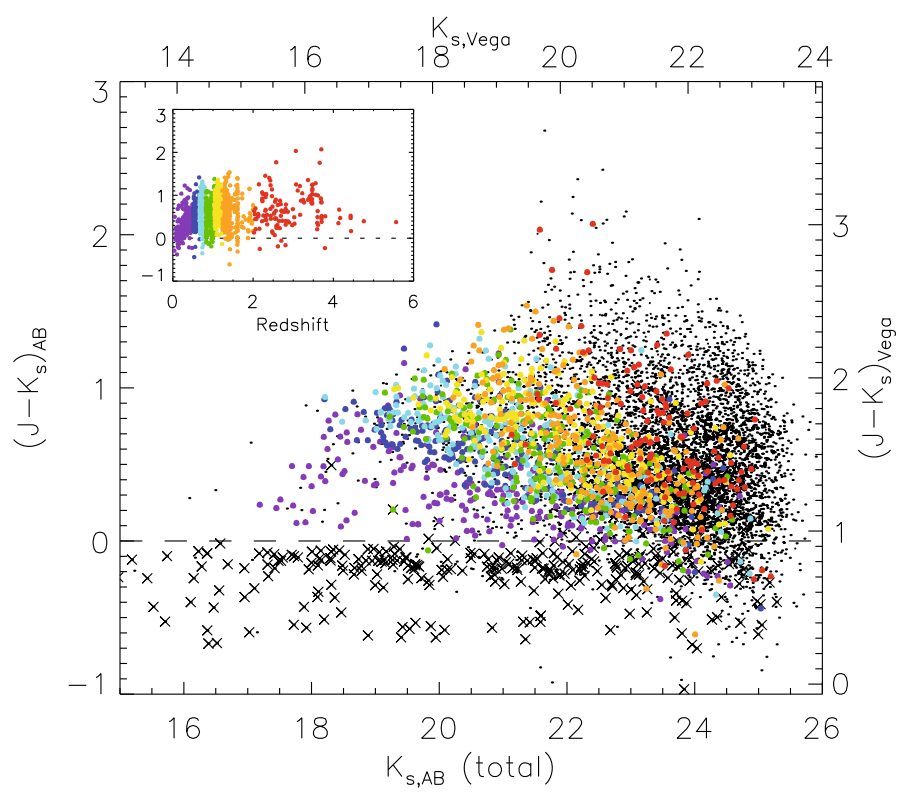

Fig. 11. NIR color-magnitude diagram (main plot) and $J-K_{\mathrm{s}}$ colorredshift distribution (inset). The color-coding is according to spectroscopic redshift using seven intervals between $0,0.5,0.67,0.82,1.04$, $1.23,2$, and 6 , as shown in the inset. Sources without spectroscopic redshift are shown as black dots. Sources which appear point-like in the HST/ACS $z$-band image are marked by crosses.

\subsection{The color catalog}

To create the $K_{\mathrm{S}}$ selected NIR color catalog, we ran SExtractor for each of the 78 tiles in double image mode with the $K_{\mathrm{s}}$ band mosaic for detection and with the respective tile for measurement using the photometric scheme described in the previous section. This approach guarantees consistent and accurate flux measurements by using unique apertures defined in $K_{\mathrm{s}}$ for all filters and by allowing to take into account the variation of the effective PSF and the sky background noise from image to image. The detections from all tiles were merged to obtain the final catalog of unique sources. In case of sources which were detected multiple times in the same band - in regions of tile overlap - the best signal-to-noise measurement evaluated based on the local limiting magnitude was kept (cf. Sect. 4.2). Eventually, differential aperture corrections were applied to the measured colors using Table 3 . The resulting catalog reports $J-K_{\mathrm{s}}$ and $H-K_{\mathrm{s}}$ and the corresponding $1 \sigma$ uncertainties for 7079 sources which have solid, i.e. $\geq 5 \sigma$, measurements in all three bands. Note that corrections for galactic extinction were not applied. The catalog data can be downloaded from the ESO archive by navigating to the URL given in the footnote on page 1 , marked by $\left(^{* *}\right)$.

The catalog columns are as follows. Columns no. 2 to 11 are directly adopted from SExtractor run on the $K_{\mathrm{s}}$ images.

(1) NUMBER - sequential source number for reference.

$(2,3)$ ALPHA_J2000, DELTA_J2000 - Source position RA, Declination (J2000).

(4) ISOAREAF_IMAGE - isophotal area in pixel units.

(5) KRON_RADIUS - Scaling of the automatic elliptical aperture inspired by Kron (1980).

(6) FWHM_IMAGE - FWHM measured in pixels units.

(7) ELLIPTICITY - Source ellipticity.

(8-10) A_WORLD, B_WORLD, THETA_WORLD Elliptical shape parameters: semi-major axis, semi-minor axis and orientation (in degree).
(11) FLAGS - SExtractor flags, possibly combined in a logical OR fashion. 1 - object has close neighbors, 2 - object was blended, 4 - object is saturated, 16 - aperture incomplete.

$(12,13)$ J_MAG_COLOR, J_MAGERR_COLOR - J magnitude and associated error from which colors are computed.

(14-17) As before but for $H$ and $K_{\mathrm{s}}$.

$(18,19)$ K_MAG_TOTAL, K_MAGERR_TOTAL - Total $K_{\mathrm{S}}$ magnitude assuming a point source profile and associated error.

$(20,21)$ J_K, J_K_ERR $-J-K_{\mathrm{s}}$ color and associated error. Differentially corrected for aperture losses.

$(22,23)$ H_K, H_K_ERR $-H-K_{\mathrm{s}}$ color and associated error.

(24) J_FRAME - Original survey tile from which the $J$ measurement was extracted. This allows to trace back to the original data if needed, for instance for the creation of image cut-outs.

$(25,26)$ As before but for $H$ and $K_{\mathrm{s}}$.

Given that aperture corrections derived for point sources are applied to mostly faint galaxies, in general, this is likely to yield an underestimate of the total flux (Col. 18), since the light profile for galaxies will be more extended than that of point sources. The amount of this effect will be quantified as a function of the extent of galaxies in Sect. 6.1.3 using image simulations.

\subsection{Color distributions}

To validate the observational data the color distribution is examined and put in perspective with redshift data taken from the literature. To this end spectroscopic redshifts were compiled from a number of published data sets, namely Cristiani et al. (2000), Croom et al. (2001), Le Fèvre et al. (2004), Strolger et al. (2004), Szokoly et al. (2004), van der Wel et al. (2004), Mignoli et al. (2005), Vanzella et al. (2008), and Popesso et al. (2009).

Figure 11 shows the $J-K_{\mathrm{s}}$ color-magnitude of $K_{\mathrm{s}}$-selected sources in the CDF-S. Owing to their bluer $J-K_{\mathrm{s}}$ color, the locus of stellar sources appears sharply offset from the bulk of redshifted galaxies which, in contrast, populate a large range of colors. In sufficiently deep observations red NIR color selection, $J-K_{\mathrm{s}}>1.34$ (corresponding to $J_{\mathrm{s}}-K_{\mathrm{s}} \gtrsim 2.3$ in the Vega system), is an efficient method to find high-redshift, i.e. $z \gtrsim 2$, galaxies (Franx et al. 2003). In our data, very red objects having $J-K_{\mathrm{s}}>1.34$ are found in the $K_{\mathrm{s}}$ range between 21 and 24.5 peaking at $K_{\mathrm{s}} \sim 22.7$. However, we note that to date there are very few spectroscopic redshifts for distant red galaxies in this field (see, e.g., Kriek et al. 2006).

The NIR color-color diagram is shown in Fig. 12 with color tracks of four Hubble-types (El, Sbc, Scd, Im) and two starburst galaxies (SB2, SB3) overlaid for which the spectral templates of Coleman et al. (1980) and two templates of starburst galaxies from Kinney et al. (1996) were redshifted to between 0.1 and 3 and folded with the actual instrumental throughput. Part of the BPZ software package of Benítez (2000) has been applied for this calculation. The smooth tracks of the El, Sbc, and Scd-types, originate from the sampling of their uniform spectral energy distribution which is dominated by the absorption-modulated continuum. In contrast, the young stellar populations of the starburst types (SB3, SB2), and to some extend also the Im-type, with their strong spectral emission features result in more wrinkled tracks.

The noticeable loop between redshift 0.3 and 0.5 , at which the $H-K_{\mathrm{s}}$ color gets bluer with increasing redshift is typical for an old stellar population and therefore most prominent for the El, Sbc and Scd types. It occurs when the restframe $K_{\mathrm{s}}$ band 


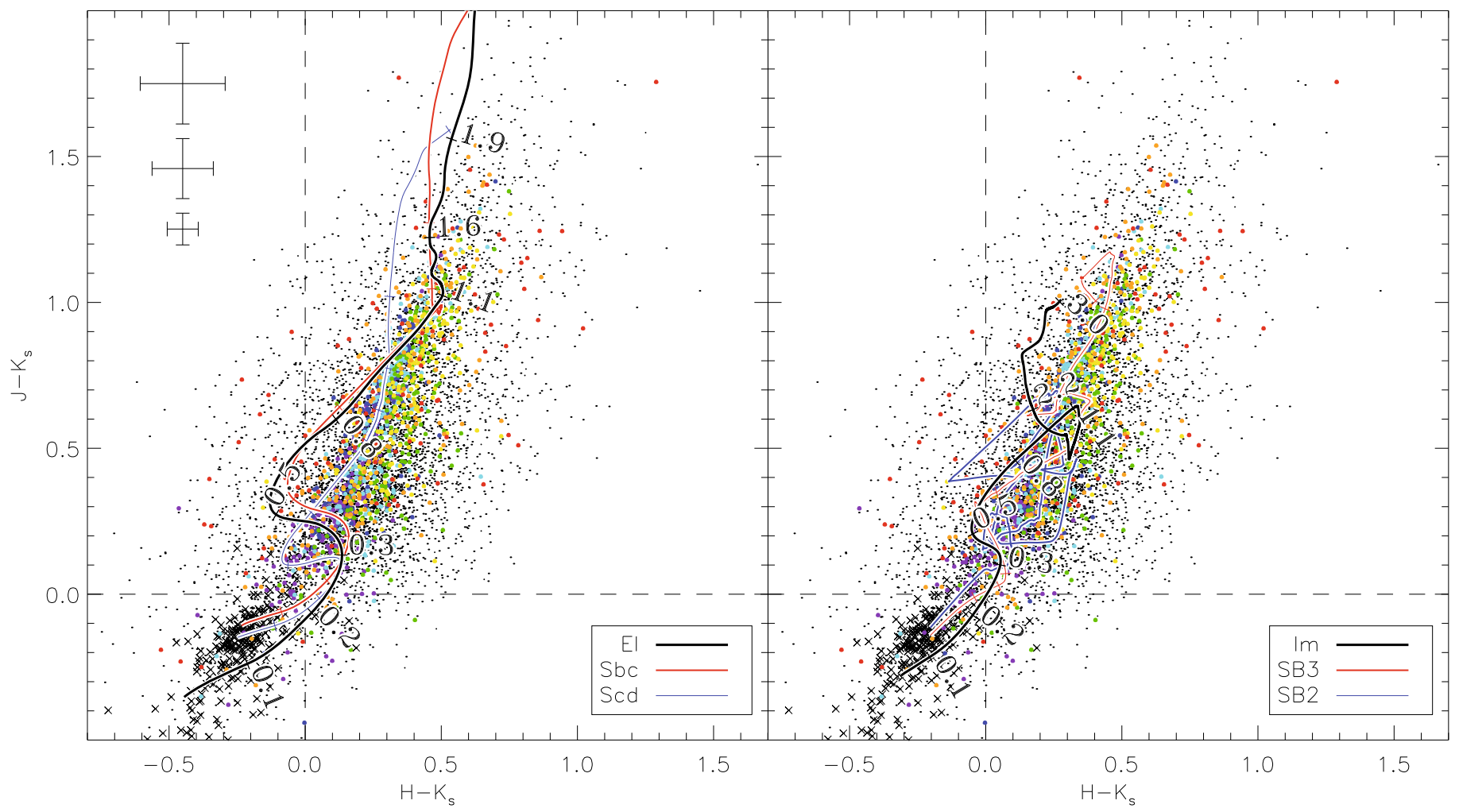

Fig. 12. Observed NIR color distribution overlaid with tracks of El, Sbc, Scd (left panel), and Im, SB3, and SB2 (right panel) galaxy spectral templates for a redshift range between 0.01 and 3 (as indicated along the curves). The color-coding according to spectroscopic redshift has been adopted from Fig. 11. Average color errors $(1 \sigma)$ being representative for sources with $K_{\mathrm{s}}=24,23,22$ are displayed top left. Sources which appear point-like in the HST/ACS $z$-band image are marked by crosses.

samples the strong absorption bands shortward of $1.5 \mu \mathrm{m}$ mainly due to TiO. For an old stellar population seen at redshifts beyond one, $J-K_{\mathrm{s}}$ is strongly increasing because the restframe $J$ flux is continuously fading due to the strong stellar atmospheric absorptions mainly induced by metals like Mg I, Fe I, Ca I etc. Towards higher redshift above $\sim 1.7$ this trend becomes even more exaggerated as the $4000-\AA /$ Balmer break starts to enter the $J$ band. The redshift dependence of the NIR colors for redshifts above 1.5 illustrates the merit of NIR data for the determination of accurate photometric redshifts when the characteristic absorption features have moved out of the optical bands. A very young stellar population seen at high redshift can not reproduce the very red NIR color as seen in the observations. Therefore, the detected very red objects can be interpreted as distant galaxies hosting an evolved stellar population. Otherwise, heavily dustabsorbed star burst galaxies could give rise to such very red colors.

\section{Analysis}

\subsection{Completeness and contamination}

\subsubsection{Empirical estimation}

Under the ESO programme 73.A-0764, led by Labbé, a very deep 21-h ISAAC $K_{\mathrm{s}}$ integration of the Hubble Ultra Deep Field (UDF) inside of the CDF-S region was obtained. The pointing is centered on $\mathrm{F} 14$ whereas the instrument was rotated by ca. 45 degrees to optimally match the Hubble UDF observations (Fig. 1). We have reduced the raw data, which are publicly available from the ESO science archive, using similar procedures as for the whole GOODS/ISAAC survey data reduction (Sect. 3). Furthermore, in order to improve the background subtraction for faint sources, we have applied a third pass in the OB processing using a source mask generated from the co-added OBs. The photometric zero points were bootstrapped from the $\mathrm{F} 14 K_{\mathrm{s}}$ image. We have co-added the calibrated OBs including the $\mathrm{F} 14 K_{\mathrm{s}}$ GOODS/ISAAC data resulting in the very deep image coving $5.40 \mathrm{arcmin}^{2}$ of the UDF region with a total integration time of $91182 \mathrm{~s}$, an effective PSF of $0.36^{\prime \prime} F W H M$, and a $5 \sigma$ depth for point sources of 25.60 mag which is 0.67 mag deeper than the respective survey image (see also Fig. 6 b).

We have utilized this image as a reference to empirically study the completeness and contamination, i.e. the fractional number of missed sources and spurious detections, in the GOODS tile F14 in the $K_{\mathrm{s}}$ band. As this is a typical tile in terms of total exposure time, image quality etc. (see Sect. 4.1), the results can be considered to be representative for the whole survey in $K_{\mathrm{s}}$ band, and - to a minor degree - for the $J$ and $H$ bands. To identify spurious detections and missed sources, we have cross-correlated the source catalogs of the two images using a $1^{\prime \prime}$ matching distance. Figure 14 displays the source counts aggregated within 0.35 -mag bins according to the total flux and the resulting completeness and contamination levels with respect to the reference image. The relative completeness was calculated from the ratio of the number of cross-identified sources and the total number of sources in the reference image both counted with respect to the total flux measured in the reference image. Hence, the resulting numbers purely represent the detection efficiency without being affected by either spurious detections or the migration effect due to flux measurement errors (Eddington bias). Counts faintward of $K_{\mathrm{s}}=24.8$ start to become significantly incomplete, dropping to a completeness of $46 \%$ at $K_{\mathrm{s}}=25.15$. At this flux level, the relative contamination which is defined as the ratio of counts of spurious sources over counts of true 

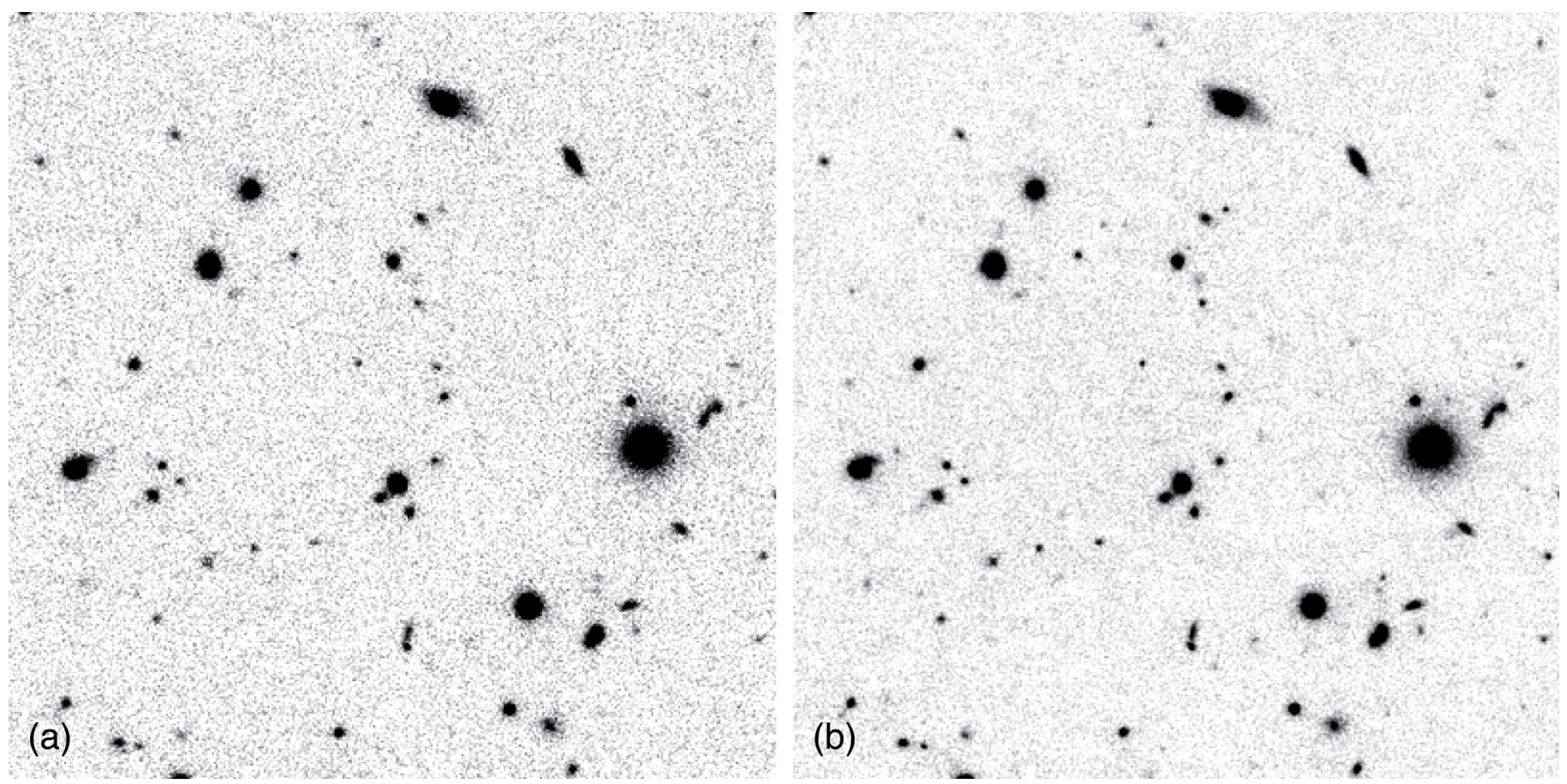

Fig. 13. Central $1^{\prime} \times 1^{\prime}$ cut-out of a) the 6-h GOODS/ISAAC survey field F14 in $K_{\mathrm{s}}$, and b) the 25.3-h co-added UDF/ISAAC $K_{\mathrm{s}}$ image, both displayed at linear scaling. The effective PSF has $0.46^{\prime \prime}$ and $0.36^{\prime \prime} F W H M$, and the $5 \sigma$ depth for point sources is 24.93 and 25.60 mag, respectively.

sources, both based on the total flux measured on F14, amounts to $26 \%$. Despite the increasing statistical uncertainty towards fainter fluxes, it is evident that the contamination becomes a dominant effect beyond the $50 \%$ completeness magnitude.

\subsubsection{Image simulations}

We have executed extensive image simulations in order to characterize the source detection efficiency i.e. the completeness level of the resulting source catalogs as a function of source flux for each tile.

The simulations were set up as follows: for each survey tile image, first, an isolated unsaturated star was selected and its $7.5^{\prime \prime} \times 7.5^{\prime \prime}$ image was cut out to create a representative template that is suitable to quantify the completeness for point sources in that tile. In order to estimate the completeness for faint galaxies, we have smoothed the stellar images using a Gaussian kernel of $0.35^{\prime \prime} F W H M$, which has been adjusted so that the distribution of central surface brightness of simulated faint objects ( 24 mag AB) matches the observational data. Although this is certainly not sufficient to simulate the observed range of galaxy types, for example low surface brightness galaxies, this approach allows the completeness to be estimated at first order without resorting to extra morphological parameters. A more accurate treatment of the statistical incompleteness taking into account a proper mix of morphological types is beyond the scope of this work but will be addressed in a forthcoming paper which will present a comparison with physical models of galaxy evolution. Then, the template image was dimmed to a flux in the magnitude range between 20 and 27 which was drawn at random from a power-law distribution whose slope of 0.26 (per magnitude) resembles the typical number counts in the field. That followed, the dimmed simulation template was co-added to the image at a random position. Per image, 500 realizations were simulated, each of which with 100 simulated sources. This corresponds to a relatively small fraction of artificial sources to real sources in the images of $1: 8.5$ on average so that the extra crowding and the statistical incompleteness due to blending induced thereby is negligible. Source detection and photometry was carried out on the simulated images in exactly the same way as for the observational data using identical detection parameters. For each tile, we have delimited the analysis to the central $75 \%$ of the image area within which the exposure time is approximately uniform. Figure $14 \mathrm{~b}$ shows that the completeness resulting from the simulations is consistent with the independent empirical estimate. This finding demonstrates the viability of the adopted simulation approach in spite of its inherent simplicity of how galaxies are modeled.

Based on the image simulations, we have characterized each tile in terms of the completeness function for point sources and for galaxies. On average the galaxy completeness is about 0.3 mag brighter than the point source completeness. The strong correlation of $m_{50}$, defined as the total flux for which the point source completeness drops to $50 \%$, with limiting magnitude can be parameterized by $m_{50} \approx m_{\text {lim }}(5 \sigma)+0.25$.

\subsubsection{Surface brightness selection}

Surface brightness selection effects are inevitable in any galaxy sample (e.g. Lilly et al. 1995) as well as the loss of flux for extended sources. To quantify the two effects, first, spheroids and disks were modelled using deVaucouleurs and exponential profiles, respectively, and were embedded into the $K_{\mathrm{s}}$ image of field F14. As a surface brightness measure, we adopt the surface brightness as inferred from the detected peak flux of each source image. Figure 15 a and $c$ show that the surface brightness serves as primary selection parameter, almost independently of total flux, source extent, and type of profile. In this case, the $90 \%$ completeness limit corresponds to a peak surface brightness of $24.0 \mathrm{mag} \operatorname{arcsec}^{-2}$, while the completeness drops below $50 \%$ for $24.5 \mathrm{mag} \mathrm{arcsec}^{-2}$. For other images, however, the numeric values are expected to vary according to image depth. Figure 15 


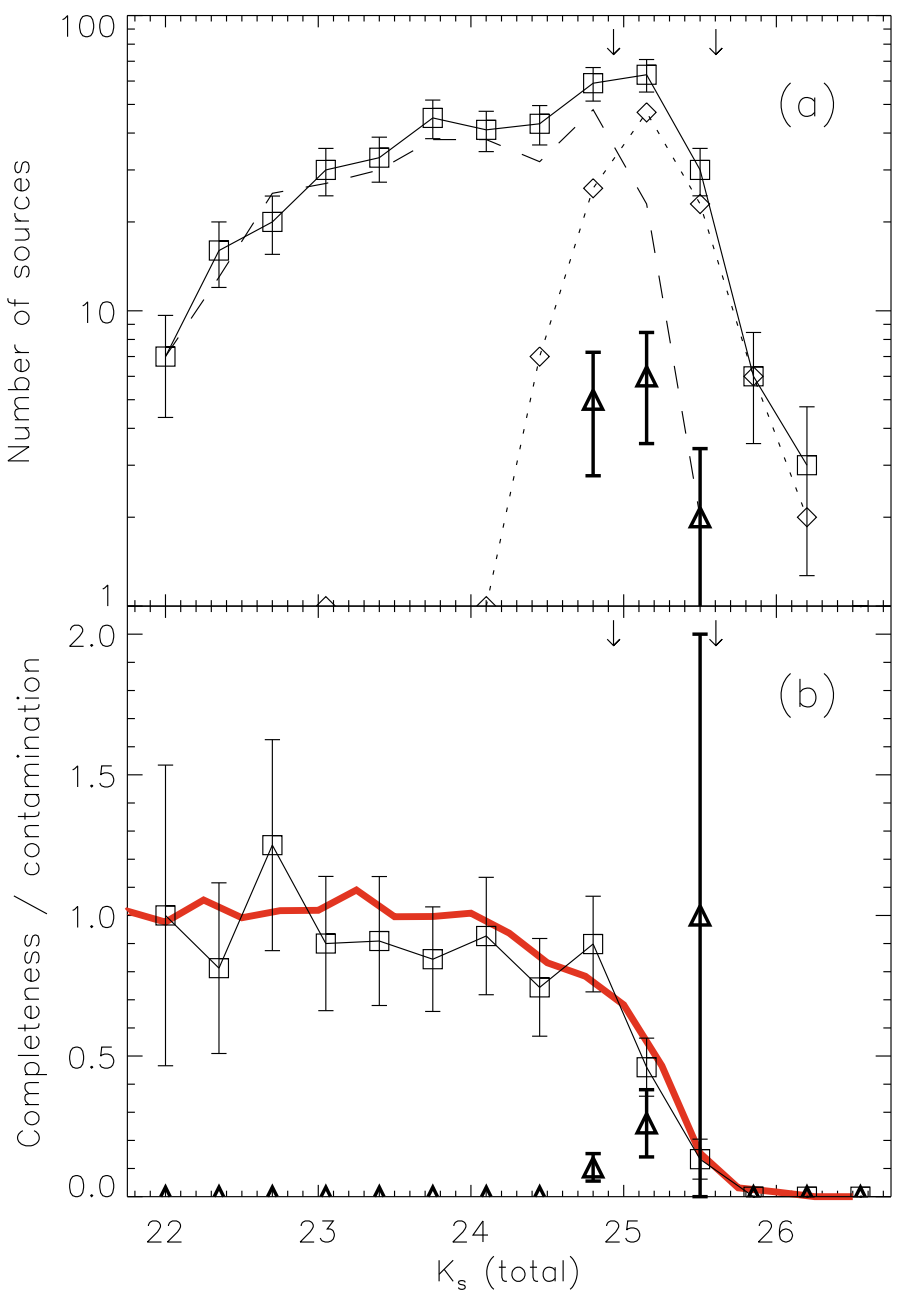

Fig. 14. a) $K_{\mathrm{s}}$ source counts in the GOODS field F14 with respect to the 0.67-mag deeper UDF/ISAAC data using exactly the same region. Plot symbols are as follows: all sources detected in the UDF/ISAAC image - squares, true positives, that is, objects cross-identified between F14 and UDF/ISAAC - dashed line, missed sources, that is, objects that are only detected in UDF/ISAAC - dotted line, and spurious detections, that is, objects that are detected in the GOODS F14 data but not in UDF/ISAAC - triangles. Error bars are Poissonian. The small arrows on the top indicate the $5 \sigma$ limiting magnitude of point sources for the two images. b) Resulting relative source completeness (squares) and relative contamination (triangles) in comparison to the completeness estimate based on image simulations (thick line, red).

$\mathrm{b}$ and d illustrate the flux loss (for MAG TOTAL) featuring a strong increase with increasing source extent. Part of the substantial flux loss of large disks is due to source blending which inhibits the proper measurement of the total flux.

The surface brightness selection effect at $24.0 \mathrm{mag} \mathrm{arcsec}^{-2}$ also becomes apparent in the observed distribution of galaxies (Fig. 16). Thanks to the deep integration of the UDF region, the properties of the sources being missed in the GOODS survey can be directly examined. In the two faintest bins in which galaxy counts are being accumulated, marked by vertical dashed lines, centered at $K_{\mathrm{s}}=24.5$ and 24.75 , the bias against low surface brightness objects is evident. Consequently, the resulting total counts in this range may be biased, but it is difficult to quantify the net effect. For objects brighter than $K_{\mathrm{s}} \sim 24.4 \mathrm{mag}$, however, there is apparently no significant selection effect.

To further examine the selection of faint galaxies depending on their physical and morphological properties, we extracted

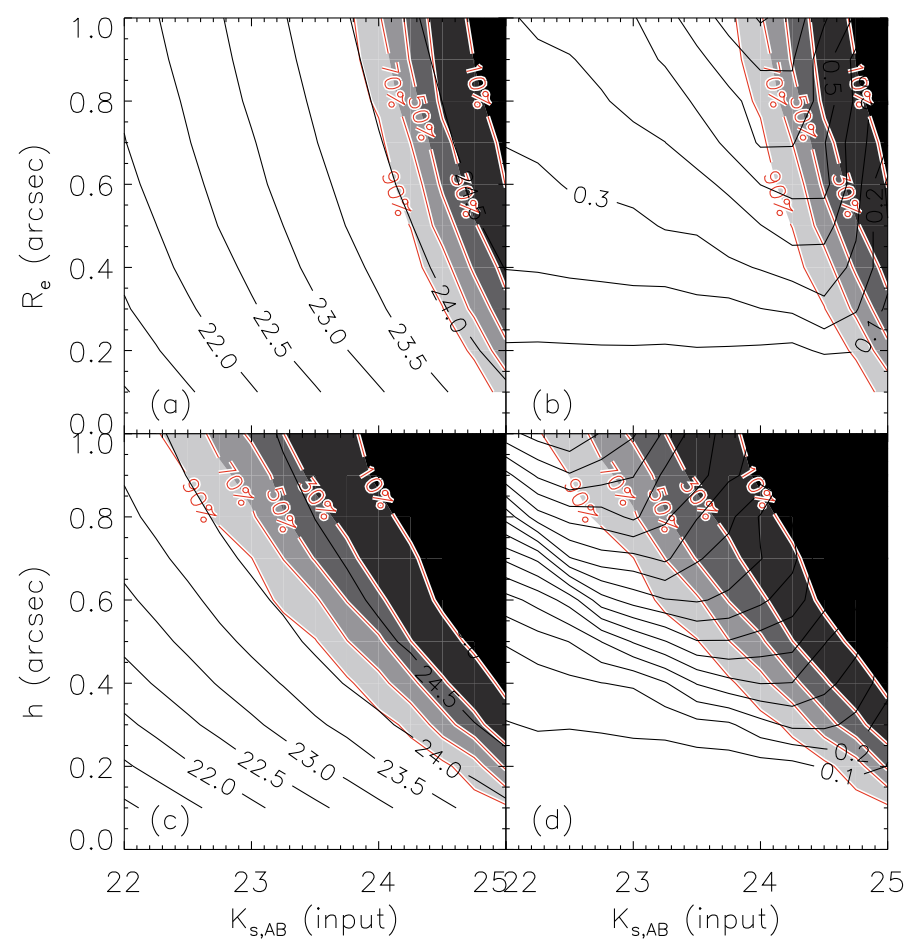

Fig. 15. Completeness, peak surface brightness and flux loss for spheroids (top panels) and disks (bottom panels) as inferred from image simulations for the GOODS field F14. $R_{\mathrm{e}}$ denotes the effective radius for spheroids and $h$ the scale length of disks. Grey shading displays the completeness in levels between $10 \%$ and $90 \%$ in each plot. Peak surface brightness (in mag $\operatorname{arcsec}^{-2}$ ) is overlaid on the left hand side a), c), and flux loss (in steps of $0.1 \mathrm{mag}$ starting from $0.1 \mathrm{mag}$ ) is shown on the right hand side b), d).

images of moderately distant objects ( $z$ between 0.15 and 0.6 ), scaled them according to redshift taking into account cosmological dimming and the $K$-correction, and computed surface brightness and flux as if they were actually observed. Figure 16 displays the resulting tracks of galaxies with the following characteristics: (a) $2.7 M^{*}$ spheroidal, (b) $0.7 M^{*}$ disk, exponentially dominated profile, (c) $0.07 M^{*}$, low-surface brightness, sub-dominant central bulge otherwise irregular morphology, (d) $2.8 M^{*}$ nearly face-on spiral, disk-dominated with central bulge. The luminous spheroidal, a, which has the most centrally concentrated profile of the sample, is least affected by surface brightness selection. In contrast, the spiral galaxy, seen nearly face-on, d, appears ca. 1.3 mag fainter in surface brightness which would be hardly detectable beyond $z=2.5$ although the luminosities of the two galaxies are similar.

\subsection{Number counts}

Differential galaxy number counts were independently determined for each of the three NIR bands as follows. We have counted the number of sources in 0.25 -mag bins with respect to the total flux (as defined in Sect. 5.1) whereas stellar sources had been excluded on the basis of their point-like appearance in the GOODS/ACS $z$-band mosaic (see below). First, each tile was processed separately and the raw counts were corrected for incompleteness according to the completeness function for galaxies which had been established using simulations (Sect. 6.1). At this point the central part of each tile, $75 \%$ in terms of area, was used over which the exposure is approximately constant. Then, for each magnitude bin the contributions from individual 


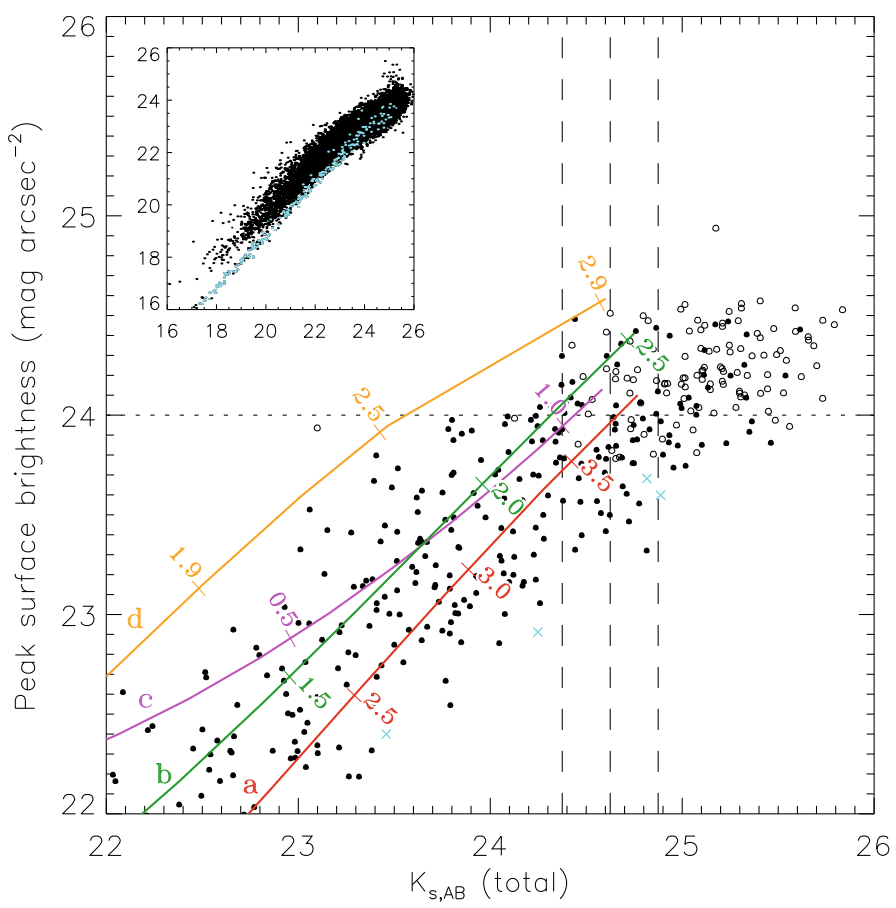

Fig. 16. Peak surface brightness as a function of $K_{\mathrm{s}}$ magnitude as defined in the text. Detections in GOODS field F14 (solid dots) opposed to missed sources (open circles) that were identified in the deeper UDF/ISAAC image. The few stars in the field are marked by cross symbols (cyan color). The insert shows the equivalent diagram for the GOODS survey as a whole with point sources being highlighted (cyan). Overlaid tracks, labelled from $a$ to $d$, correspond to individual galaxies as if they were observed at the redshifts denoted along the curves.

tiles having at least $50 \%$ point source completeness were combined. In this way the variance of the effective area as a function of depth was accounted for. The correction factor is effectively bound and, consequently, only the deepest tiles contribute to the faintest bins (which are $J$ and $K_{\mathrm{s}}=25.25$ and $H=25.0$ ). The actually applied completeness correction factors are about 1.6 for the faintest GOODS/CDF-S data points in all three bands, and 1.7 for the data point at $K_{\mathrm{s}}=25.25$ which was extracted from the UDF/ISAAC image. The resulting differential counts are displayed in Fig. 17 and reported in Table 4 where cumulative counts, $N(<m)$, are quoted in addition.

As identification criterion for stars, a cut in $R_{50}$ was used in conjunction with the flux limit $z_{\mathrm{AB}} \leq 24.5$ (MAG_AUTO) to restrict the selection to the flux range where the stellarity criterion is able to clearly identify the stellar locus. Then, it was verified that all sources have $J-K_{\mathrm{s}}<0$, which is a very good criterion for the whole range of stellar types except for the very cool but comparatively rare $\mathrm{L}$ and $\mathrm{T}$ dwarf types (e.g. Hawley et al. 2002), leading to the removal of 5 objects most of which have been independently spectroscopically identified as QSO (cf. Fig. 11). The number counts of the resulting sample of 295 stars are perfectly concordant with the Galaxy model as simulated by Girardi et al. (2005) (Fig. 17, right panel). Beyond $K_{\mathrm{s}} \sim 24$, the sample becomes incomplete due to the imposed flux limit in $z$ band, as expected. Furthermore, using the angular correlation function, we have checked that the magnitude-limited stellar sample $\left(17<K_{\mathrm{S}}<23\right)$ is uncorrelated over the full range of angular scales probed by the survey $\left(\theta \sim 0.0005 \ldots 0.1^{\circ}\right)$, which, besides, confirms the photometric homogeneity of the data. Altogether, the tests provide no evidence for a significant contamination of the stellar sample from e.g. compact galaxies.
Thus, the resulting galaxy number counts are simply affected by the incomplete subtraction of stellar sources beyond $K_{\mathrm{s}} \sim 24$. The amplitude of this effect, however, is just about $2 \%$ which is less than the size of the plot symbols in Fig. 17.

The faint-end galaxy number counts $(m \geq 22)$ follow an exponential with logarithmic slope of $\alpha=(0.262 \pm 0.007) \mathrm{mag}^{-1}$ for $J$ and, marginally shallower, $\alpha=(0.254 \pm 0.008) \mathrm{mag}^{-1}$, for $H$ and $K_{\mathrm{s}}$. The best-fitting amplitudes (in a least squares sense) are 3.31, 5.77, and $6.40 \times 10^{-2} \mathrm{mag}^{-1} \mathrm{deg}^{-2}$ for $J, H$, and $K_{\mathrm{s}}$, respectively. Down to the faintest fluxes we do not find an indication for a significant change in the slope of the number counts. According to the size of the survey area which exceeds previous surveys of similar depth by about an order of magnitude, the statistical errors turn out to be almost negligible over a large range of fluxes. At the faintest fluxes, the statistical errors of the data points must slightly increase because of the decrease of the effective area. Generally, the results in the low-flux regime may be systematically affected by uncertainties in the incompleteness correction and possible contamination due to spurious detections. To minimize the possible impact, we have limited the analysis to $\gtrsim 5 \sigma$ significant sources. As a consequence of this choice, correction factors are quite moderate and the contamination level remains low $(\sim 25 \%)$.

Comparing our results with previously published data of deep surveys selected from the literature, we find a good agreement in general (Fig. 17). However, closer examination reveals some discrepancies much in the same way as discrepancies have been found amongst previous data sets (e.g. Saracco et al. 2001; Metcalfe et al. 2006). For instance, Saracco et al. (2001) reported, based on the deep imaging of the F16 field in the CDF$S$ region, comparatively steep $J$-band number counts with systematically more faint $(J>24)$ galaxies than we have found. By contrast the number counts resulting from the Subaru Deep Survey (Maihara et al. 2001) are consistent with our results in the $J$-band (and similarly in the $K_{\mathrm{s}}$-band), likewise the raw number counts in the Hubble Deep Field-North obtained with HST/NICMOS using the $F 110 W$ filter (Thompson et al. 1999). In the $H$-band the results of two imaging surveys which have been conducted in the CDF-S region previously, by Moy et al. (2003) and Cimatti et al. (2002), are fully compatible with our findings. Also the number counts resulting from the completely independent study by Metcalfe et al. (2006) based on imaging of the $7^{\prime} \times 7^{\prime}$ William Herschel Deep Field using the Omega Prime camera on the 3.5-m Calar Alto telescope, Spain, are perfectly in agreement with our data. The extremely deep $H$-band number counts reported by Metcalfe et al. (2006) based on observations of the Hubble Deep Field-South (HDF-S) using NICMOS seem to be slightly in excess with respect to our findings, however, taking into account the small size of the HDF-S, $0.95 \times 0.95 \operatorname{arcmin}^{2}$, the two results are consistent within the $1 \sigma$ Poissonian errors. Our $K_{\mathrm{s}}$-band results are compatible with the UKIDSS UDS, that is the ultra-deep survey of the suite of infrared deep sky surveys using the United Kingdom Infrared Telescope (Hartley et al. 2008), the faint infrared extragalactic survey (FIRES) in the MS 1054-03 field and in the HDF-S (Förster Schreiber et al. 2006; Labbé et al. 2003), the K20 survey Cimatti et al. (2002), the $K_{\mathrm{s}}$-band observations of Saracco et al. (2001) in the CDF-S and the Subaru Deep Survey (Maihara et al. 2001) once the formal uncertainties are taken into account. Concerning the faint end of the $K_{\mathrm{s}}$ counts, though individual data points are formally consistent within the error bars, the slope that we find is steeper than what has been reported for the FIRES survey, namely $\alpha \approx 0.15$ by Labbé et al. (2003), and $\alpha=0.20$ by Förster Schreiber et al. (2006). However, the comparison of 


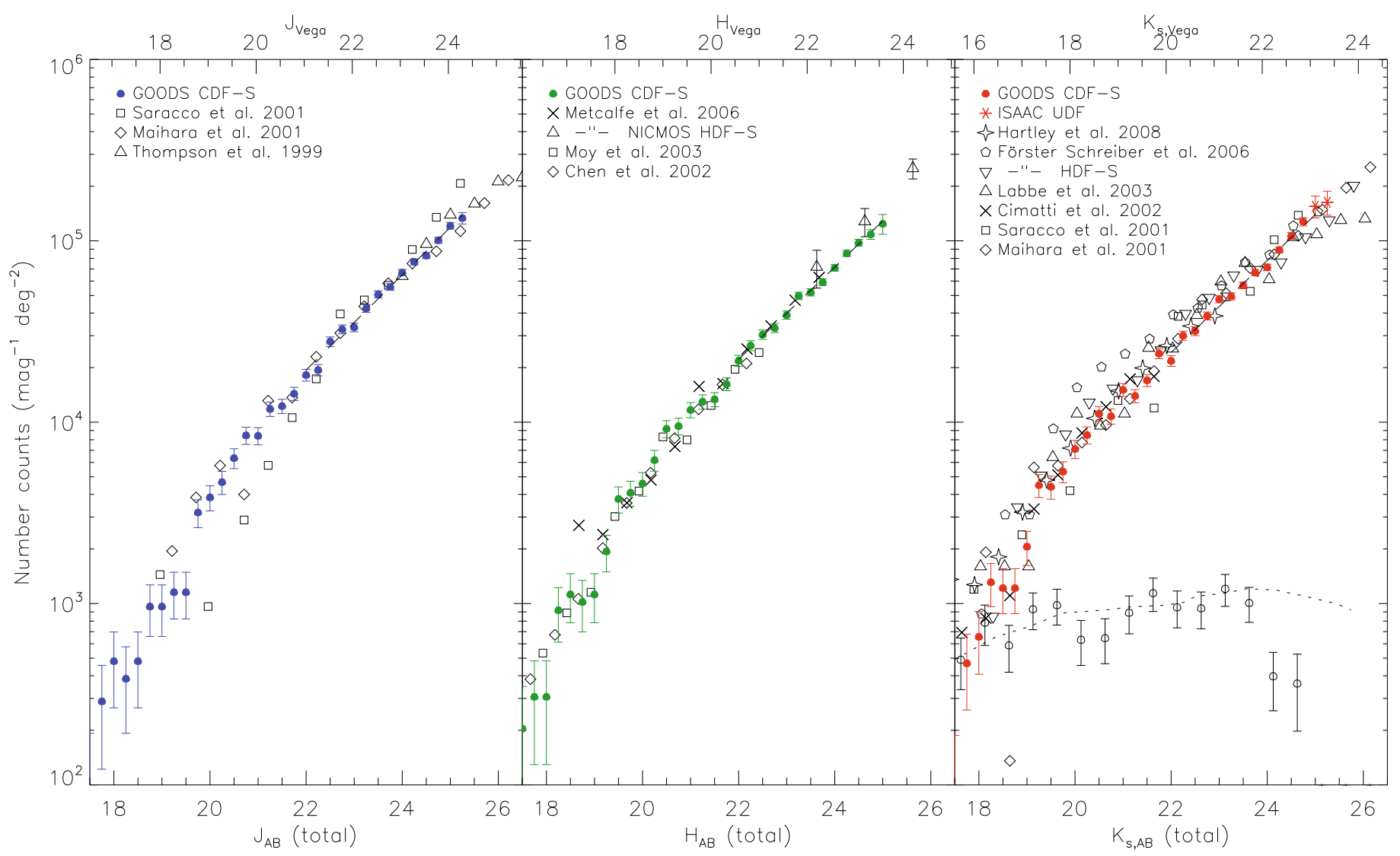

Fig. 17. Differential galaxy number counts in the GOODS/CDF-S region in the $J, H$, and $K_{\mathrm{s}}$ bands (left, middle, and right panel, respectively) corrected for incompleteness and displayed with Poissonian $(1 \sigma)$ error bars. The two faintest data points in $K_{\mathrm{s}}$ (asterisk symbols) were inferred from the very deep UDF/ISAAC image. The dashed lines indicate the faint-end exponential fits with logarithmic slopes between 0.24 and $0.27 \mathrm{mag}^{-1}$ (see text). Results from other NIR surveys selected from the literature are displayed for comparison. Generally, incompleteness-corrected data is shown except for the HST/NICMOS results for which incompleteness at the depth of the GOODS/ISAAC data is negligible. Note that the data labeled Saracco et al. (2001) refers to the results obtained from the CDF-S, the data of Thompson et al. (1999) shown here is limited to $J \geq 24$ for which the relatively small field provides a sufficient absolute number of counts. The apparent bump around $K_{\mathrm{s}} \sim 20$ in the data of Förster Schreiber et al. (2006) is owing to the contribution from the MS 1054-03 cluster of galaxies on which the survey field is centered and should not be seen as discrepancy with the other data sets. The $K_{\mathrm{s}}$ data from Förster Schreiber et al. (2006) HDF-S refers to the results of Labbé et al. (2003) but it is apparently not identical, however, as to the comparison with our results this incongruity seems to be irrelevant. In the right panel, the stellar $K_{\mathrm{s}}$-counts in the GOODS/CDF-S (circles) are shown in contrast to the simulation of Girardi et al. (2005) based on a model of the Galaxy (dotted line).

number counts results must be exercised with caution. At faint flux levels, close to the limiting magnitude of the surveys, the statistical corrections and their difficult to assess uncertainties usually dominate the error budget. In general, any photometric mismatch, e.g. due to discrepancies in the passband, the details of the flux measurement scheme, or the correction to total flux, may result in differences of the number counts. Furthermore, the presence of cosmic large-scale structure (LSS) causes a variation of number counts from field to field in excess to the usually quoted fluctuation according to Poissonian statistics.

To quantify the effect of cosmic variance on our results, we have assumed an angular correlation of power-law form with the canonical index, i.e. $w(\theta)=A \theta^{-0.8}$, and a correlation amplitude based on the measured angular clustering (see, e.g., Daddi et al. 2003). Taking into account the actual survey area, we find the fractional cosmic variance of the cumulative counts for $m<22,23$, and $24 \mathrm{mag}(\mathrm{AB})$ to be $3.4,3.2$, and $3.0 \%(1 \sigma)$, respectively, in addition to the respective Poissonian fluctuations of $2.5,1.8$, and $1.7 \%$. Put differently, cosmic variance increases the Poissonian errors on the (cumulative) number counts by factors between $\sim 1.7$ and 2.1. According to the power-law model, Poissonian fluctuations decrease more rapidly with increasing survey area $(\Omega)$ than the LSS fluctuations, namely $\propto \Omega^{-0.5}$ vs. $\propto \Omega^{-0.2}$. Therefore, the contribution of cosmic variance to the total error budget will be significant also for next generation deep surveys. For number counts down to $24 \mathrm{mag}$ in a $1^{\circ} \times 1^{\circ}$ field, for instance, one expects about $0.34 \%$ Poissonian and $1.6 \%$ LSS fluctuations.

\section{Summary}

We presented the deep VLT/ISAAC NIR imaging survey in the GOODS-South field. The survey was conducted as part of the ESO/GOODS program and the resulting data products were released to the public via the ESO science archive. The survey covers an area of $173 \operatorname{arcmin}^{2}$ in $J$ and $K_{\mathrm{s}}$, and $160 \operatorname{arcmin}^{2}$ in the $H$ band. The "median" survey depth is 25.1 in $J$ and 24.7 both in $H$ and $K_{\mathrm{s}}$ in terms of the $5 \sigma$ total limiting $A B$ magnitude for point sources. The excellent image quality is characterized by a PSF with FWHM between $0.34^{\prime \prime}$ and $0.65^{\prime \prime}$. The astrometric calibration is accurate to $\sim 0.06^{\prime \prime}$ RMS over the whole field. The absolute astrometric accuracy is limited by the accuracy of the GSC 2 on which the astrometry is ultimately based. The photometric calibration was verified in various ways including 
J. Retzlaff et al.: The Great Observatories Origins Deep Survey

Table 4. Galaxy number counts in $J, H$, and $K_{\mathrm{s}}$ as function of total magnitude, $m$, expressed in the AB photometric system.

\begin{tabular}{|c|c|c|c|c|c|c|c|c|c|}
\hline \multirow[b]{2}{*}{ Mag. } & \multicolumn{3}{|c|}{$J$} & \multicolumn{3}{|c|}{$H$} & \multicolumn{3}{|c|}{$K_{\mathrm{s}}$} \\
\hline & $n(m)$ & $\Delta n$ & $N(<m)$ & $n(m)$ & $\Delta n$ & $N(<m)$ & $n(m)$ & $\Delta n$ & $N(<m)$ \\
\hline 17.00 & - & - & - & - & - & - & 187.4 & 132.5 & 46.8 \\
\hline 17.25 & 192.6 & 136.2 & 48.1 & 204.1 & 144.3 & 51.0 & 93.7 & 93.7 & 70.3 \\
\hline 17.50 & 96.3 & 96.3 & 72.2 & 204.1 & 144.3 & 102.1 & 93.7 & 93.7 & 93.7 \\
\hline 17.75 & 288.9 & 166.8 & 144.4 & 306.2 & 176.8 & 178.6 & 468.4 & 209.5 & 210.8 \\
\hline 18.00 & 481.5 & 215.3 & 264.8 & 306.2 & 176.8 & 255.1 & 655.8 & 247.9 & 374.7 \\
\hline 18.25 & 385.2 & 192.6 & 361.1 & 918.5 & 306.2 & 484.7 & 1311.5 & 350.5 & 702.6 \\
\hline 18.50 & 481.5 & 215.3 & 481.5 & 1122.6 & 338.5 & 765.4 & 1217.9 & 337.8 & 1007.1 \\
\hline 18.75 & 962.9 & 304.5 & 722.2 & 1020.5 & 322.7 & 1020.5 & 1217.9 & 337.8 & 1311.5 \\
\hline 19.00 & 962.9 & 304.5 & 962.9 & 1122.6 & 338.5 & 1301.2 & 2061.0 & 439.4 & 1826.8 \\
\hline 19.25 & 1155.5 & 333.6 & 1251.8 & 1939.0 & 444.8 & 1785.9 & 4496.7 & 649.0 & 2951.0 \\
\hline 19.50 & 1155.5 & 333.6 & 1540.7 & 3775.9 & 620.8 & 2729.9 & 4403.0 & 642.2 & 4051.7 \\
\hline 19.75 & 3177.7 & 553.2 & 2335.1 & 4082.1 & 645.4 & 3750.4 & 5339.8 & 707.3 & 5386.7 \\
\hline 20.00 & 3851.7 & 609.0 & 3298.0 & 4592.3 & 684.6 & 4898.5 & 7119.8 & 816.7 & 7166.6 \\
\hline 20.25 & 4674.1 & 680.9 & 4466.6 & 6181.6 & 807.0 & 6443.9 & 8497.0 & 913.4 & 9290.9 \\
\hline 20.50 & 6343.3 & 793.3 & 6052.4 & 9204.2 & 992.4 & 8744.9 & 11152.5 & 1045.0 & 12079.0 \\
\hline 20.75 & 8464.5 & 921.1 & 8168.5 & 9517.3 & 1002.5 & 11124.2 & 10786.6 & 1030.8 & 14775.7 \\
\hline 21.00 & 8417.2 & 914.9 & 10272.8 & 11691.1 & 1116.5 & 14047.0 & 15092.2 & 1224.9 & 18548.7 \\
\hline 21.25 & 11834.9 & 1081.4 & 13231.6 & 12978.5 & 1178.5 & 17291.7 & 13970.6 & 1169.0 & 22041.4 \\
\hline 21.50 & 12273.7 & 1111.6 & 16300.0 & 13363.6 & 1191.6 & 20632.6 & 17009.5 & 1297.3 & 26293.7 \\
\hline 21.75 & 14373.0 & 1199.2 & 19893.2 & 16229.6 & 1319.8 & 24690.0 & 23914.1 & 1535.0 & 32272.3 \\
\hline 22.00 & 18201.4 & 1357.0 & 24443.6 & 21834.4 & 1536.5 & 30148.6 & 21793.7 & 1465.3 & 37720.7 \\
\hline 22.25 & 19396.3 & 1396.6 & 29292.7 & 26466.7 & 1684.3 & 36765.2 & 30044.2 & 1729.9 & 45231.7 \\
\hline 22.50 & 27849.6 & 1685.1 & 36255.1 & 30430.8 & 1799.7 & 44372.9 & 31809.6 & 1772.8 & 53184.1 \\
\hline 22.75 & 32572.9 & 1824.4 & 44398.3 & 33121.6 & 1887.9 & 52653.4 & 38564.6 & 1939.3 & 62825.3 \\
\hline 23.00 & 33383.1 & 1839.5 & 52744.1 & 39008.2 & 2042.3 & 62405.4 & 47694.9 & 2167.0 & 74749.0 \\
\hline 23.25 & 42430.3 & 2076.1 & 63351.6 & 49751.0 & 2312.7 & 74843.1 & 49461.9 & 2207.0 & 87114.5 \\
\hline 23.50 & 50740.0 & 2278.4 & 76036.6 & 52058.2 & 2372.2 & 87857.7 & 56856.0 & 2378.3 & 101328.5 \\
\hline 23.75 & 55735.1 & 2396.4 & 89970.4 & 59219.8 & 2549.0 & 102662.7 & 66811.4 & 2649.3 & 118031.4 \\
\hline 24.00 & 66881.7 & 2623.6 & 106690.8 & 70936.5 & 2903.4 & 120396.8 & 71444.1 & 2870.9 & 135892.4 \\
\hline 24.25 & 76601.7 & 2880.8 & 125841.2 & 85300.8 & 3424.7 & 141722.0 & 89161.3 & 3459.9 & 158182.7 \\
\hline 24.50 & 82958.4 & 3124.9 & 146580.9 & 97756.2 & 4236.4 & 166161.0 & 106466.9 & 4435.9 & 184799.5 \\
\hline 24.75 & 100728.4 & 3775.7 & 171763.0 & 108646.9 & 6676.6 & 193322.8 & 127600.9 & 6680.3 & 216699.7 \\
\hline 25.00 & 121070.0 & 5210.8 & 202030.5 & 124232.9 & 15534.6 & 224381.0 & 155102.4 & 21395.0 & 255475.3 \\
\hline 25.25 & 133487.5 & 9676.3 & 235402.3 & - & - & - & 163124.3 & 24634.1 & 296256.3 \\
\hline
\end{tabular}

Notes. Differential counts and $1 \sigma$-errors, $n(m) \pm \Delta n$, are quoted per unit mag per square degree. Cumulative counts, $N(<m)$, are per square degree.

tests against independent NIR photometric measurements such as 2MASS. The internal accuracy of the photometric calibration is $\leq 0.017 \mathrm{mag}(1 \sigma)$. The overall photometric accuracy, including systematic effects, is up to $\sim 0.05$ mag for all three bands. We described the survey layout, observations, data reduction and calibration, and the quality control process. The data products were characterized in terms of PSF width, curve of growth, and limiting depth. A $K_{\mathrm{s}}$-selected catalog of 7079 objects sources with $J H K_{\mathrm{s}}$ photometry was defined for which color distributions were presented with respect to available redshift data.

We characterized the properties of extracted source catalogs in terms of their fractional completeness and contamination as a function of magnitude. For this purpose additional $21 \mathrm{~h}$ of $K_{\mathrm{s}}$ imaging data of the Hubble Ultra Deep field, which are available from the ESO archive, were processed and the resulting image, which is $\sim 0.7$ mag deeper than the respective survey image, was analyzed. These results were combined with extensive image simulations. For the number counts of faint galaxies we found very similar logarithmic slopes in all three bands, ranging at the faint end between 0.24 and $0.27 \mathrm{mag}^{-1}$ without an indication for a change in the slope. Our new results are largely compatible with the ones from previous surveys. However, the GOODS/ISAAC data establish the surface density of faint galaxies in the Universe with unprecedented accuracy owing to its unique combination of survey area and depth which is unrivaled by any other NIR survey. In fact, this survey represents the deepest NIR imaging data set to date covering a contiguous region well above $100 \operatorname{arcmin}^{2}$. In the $H$ band, there is to our knowledge no ground based survey paralleling GOODS/ISAAC in depth.

Acknowledgements. This long term large program would have not been successful without the dedication and excellent support of the ESO Paranal staff. We are also grateful to F. Comeron and the ESO User Support group for their continuous assistance in scheduling the observations and monitoring their status.

\section{References}

Amico, P., Cuby, J., Devillard, N., Jung, Y., \& Lidman, C. 2002, ISAAC Data Reduction Guide 1.5, European Organisation for Astronomical Research in the Southern Hemisphere

Benítez, N. 2000, ApJ, 536, 571

Bertin, E., \& Arnouts, S. 1996, A\&AS, 117, 393

Carpenter, J. M. 2001, AJ, 121, 2851

Chen, H.-W., McCarthy, P. J., Marzke, R. O., et al. 2002, ApJ, 570, 54

Cimatti, A., Mignoli, M., Daddi, E., et al. 2002, A\&A, 392, 395 Coleman, G. D., Wu, C.-C., \& Weedman, D. W. 1980, ApJS, 43, 393

Cowie, L. L., Gardner, J. P., Hu, E. M., et al. 1994, ApJ, 434, 114 Cristiani, S., Appenzeller, I., Arnouts, S., et al. 2000, A\&A, 359, 489 Croom, S. M., Warren, S. J., \& Glazebrook, K. 2001, MNRAS, 328, 150

Daddi, E., Röttgering, H. J. A., Labbé, I., et al. 2003, ApJ, 588, 50

Daddi, E., Cimatti, A., Renzini, A., et al. 2004, ApJ, 617, 746

Dickinson, M., Giavalisco, M., \& The Goods Team. 2003, in The Mass of Galaxies at Low and High Redshift, ed. R. Bender, \& A. Renzini, 324 Förster Schreiber, N. M., Franx, M., Labbé, I., et al. 2006, AJ, 131, 1891 Franx, M., Labbé, I., Rudnick, G., et al. 2003, ApJ, 587, L79

Giavalisco, M. 2005, New Astron. Rev., 49, 440 
Giavalisco, M., Ferguson, H. C., Koekemoer, A. M., et al. 2004, ApJ, 600, L93 Girardi, L., Groenewegen, M. A. T., Hatziminaoglou, E., et al. 2005, A\&A, 436, 895

Hartley, W. G., Lane, K. P., Almaini, O., et al. 2008, MNRAS, 391, 1301

Hawarden, T. G., Leggett, S. K., Letawsky, M. B., Ballantyne, D. R., \& Casali, M. M. 2001, MNRAS, 325, 563

Hawley, S. L., Covey, K. R., Knapp, G. R., et al. 2002, AJ, 123, 3409

Hunt, L. K., Mannucci, F., Testi, L., et al. 1998, AJ, 115, 2594

Kinney, A. L., Calzetti, D., Bohlin, R. C., et al. 1996, ApJ, 467, 38

Koranyi, D. M., Kleyna, J., \& Grogin, N. A. 1998, PASP, 110, 1464

Kriek, M., van Dokkum, P. G., Franx, M., et al. 2006, ApJ, 649, L71

Kron, R. G. 1980, ApJS, 43, 305

Labbé, I., Franx, M., Rudnick, G., et al. 2003, AJ, 125, 1107

Le Fèvre, O., Vettolani, G., Paltani, S., et al. 2004, A\&A, 428, 1043

Lilly, S. J., Le Fevre, O., Crampton, D., Hammer, F., \& Tresse, L. 1995, ApJ, 455,50

Maihara, T., Iwamuro, F., Tanabe, H., et al. 2001, PASJ, 53, 25

Mason, E., Lombardi, G., Lidman, C., et al. 2008, in The 2007 ESO Instrument Calibration Workshop, ed. A. Kaufer \& F. Kerber (Springer-Verlag Berlin Heidelberg), 439

Metcalfe, N., Shanks, T., Weilbacher, P. M., et al. 2006, MNRAS, 370, 1257
Mignoli, M., Cimatti, A., Zamorani, G., et al. 2005, A\&A, 437, 883

Moorwood, A., Cuby, J.-G., \& Lidman, C. 1998, The Messenger, 91, 9

Moorwood, A., Cuby, J.-G., Ballester, P., et al. 1999, The Messenger, 95, 1

Moy, E., Barmby, P., Rigopoulou, D., et al. 2003, A\&A, 403, 493

Oke, J. B., \& Gunn, J. E. 1983, ApJ, 266, 713

Olsen, L. F., Miralles, J.-M., da Costa, L., et al. 2006, A\&A, 452, 119

Persson, S. E., Murphy, D. C., Krzeminski, W., Roth, M., \& Rieke, M. J. 1998, AJ, 116, 2475

Popesso, P., Dickinson, M., Nonino, M., et al. 2009, A\&A, 494, 443

Renzini, A., Cesarsky, C., Cristiani, S., et al. 2003, in The Mass of Galaxies at Low and High Redshift, ed. R. Bender, \& A. Renzini, 332

Saracco, P., Giallongo, E., Cristiani, S., et al. 2001, A\&A, 375, 1

Skrutskie, M. F., Cutri, R. M., Stiening, R., et al. 2006, AJ, 131, 1163

Strolger, L.-G., Riess, A. G., Dahlen, T., et al. 2004, ApJ, 613, 200

Szokoly, G. P., Bergeron, J., Hasinger, G., et al. 2004, ApJS, 155, 271

Thompson, R. I., Storrie-Lombardi, L. J., Weymann, R. J., et al. 1999, AJ, 117, 17

van der Wel, A., Franx, M., van Dokkum, P. G., et al. 2004, ApJ, 601, L5

Vandame, B. 2004, Processing Multi-wavelength and Wide-Field Imaging data: Application to the ESO Imaging Survey (Nice University)

Vanzella, E., Cristiani, S., Dickinson, M., et al. 2008, A\&A, 478, 83 